\title{
Existência e concentração de soluções para equações de Schrödinger ${ }^{1}$
}

\author{
Alex Eduardo Andrade Borges \\ Orientador: Prof. Dr. Sérgio Henrique Monari Soares
}

Dissertação apresentada ao Instituto de Ciências Matemáticas e de Computação da Universidade de São Paulo, como parte dos requisitos para obtenção do título de Mestre em ciências - Área: Matemática.

USP - São Carlos

Fevereiro/2004

${ }^{1}$ Este trabalho teve suporte financeiro da CAPES 


\section{A Comissão Julgadora:}

Prof. Dr. Sérgio Henrique Monari Soares

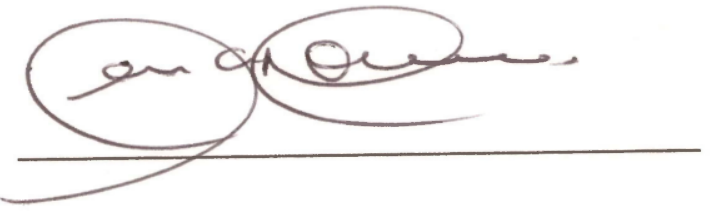

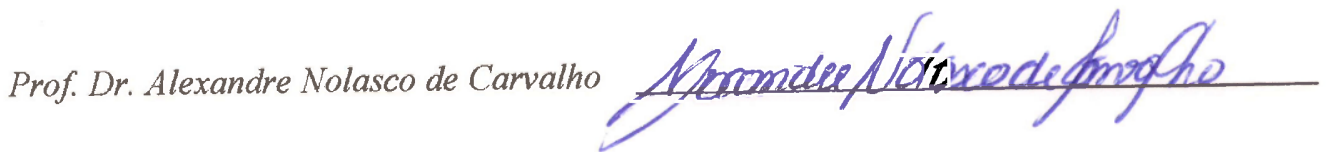

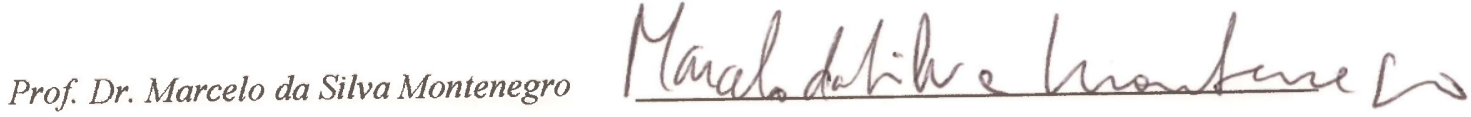


Aos meus pais, esposa e filho, com amor... 


\section{Agradecimentos}

Acima de tudo a Deus, por estar sempre ao meu lado.

Aos meus pais, Carlos e Ninha, pelo amor, preocupação e apoio que sempre manifestaram e principalmente pelo esforço que fizeram para que eu tivesse oportunidade de estudo e conseguisse concluir mais esta etapa da minha vida.

À amada esposa Vânia, por ter sido amante e companheira no decorrer deste percurso tão cheio de surpresas.

Ao meu pequenino Luís Felipe, por ter sido uma das surpresas maravilhosas da minha vida.

Ao Prof. Sérgio Henrique Monari Soares, pela orientação, dedicação, paciência, amizade e seriedade profissional com que conduziu este trabalho.

Aos meus irmãos e familiares que acreditaram e torceram por mim.

À turma de matemática/98 da USP e aos amigos da pós graduação, que sempre me incentivaram, me ajudaram de alguma forma ultrapassar muitos obstáculos e tornaram esta caminhada mais suave.

À todos os professores e funcionários do ICMC, que de muitas formas contribuiram para a realização deste trabalho, em especial aos Professores Valdir e Janete pelos anos de orientação em iniciação científica.

Enfim, a todos aqueles que colaboraram de alguma forma para a realização deste trabalho.

Muito Obrigado! 


\section{Resumo}

O objetivo deste trabalho é estudar a existência e o comportamento de concentração de ondas estacionárias para a equação de Schrödinger não linear

$$
i h \frac{\partial \psi}{\partial t}=-\frac{h^{2}}{2 m} \Delta \psi+V(x) \psi-\gamma|\psi|^{p-1} \psi .
$$

Sob diferentes hipóteses em $V$, várias condições suficientes para a existência de soluções não triviais são estabelecidas. Em particular, quando $V$ satisfaz uma certa condição no infinito, é provado que essas soluções se concentram em pontos de mínimo global de $V$ quando $h \rightarrow 0$. 


\section{Abstract}

The aim of this work is to study the existence and the concentration behavior of standing wave solutions of the nonlinear Schrödinger equation

$$
i h \frac{\partial \eta}{\partial t}=-\frac{h^{2}}{2 m} \Delta \psi+V(x) \psi-\gamma|\psi|^{p-1} \psi .
$$

Making different assumptions on $V$, various sufficient conditions for the existence of nontrivial solutions are established. In particular, under certain condition on $V$ at infinity, it is proved that these solutions concentrate at global minimum points of $V$ as $h \rightarrow 0$. 


\section{Sumário}

Introdução

1 Existência de Soluções para Equações de Schrödinger com Coeficientes Periódicos

2 Existência de Soluções para Equações de Schrödinger

3 Concentração de Soluções de Equações de Schrõdinger

A Diferenciabilidade do Funcional Associado 42

B Teorema do Passo da Montanha

C Princípio Variacional de Ekeland 48

D Lema de Lions

Referências Bibliográficas 


\section{Introdução}

Neste trabalho, estudamos alguns resultados de existência de soluções para equações elípticas não lineares em $\mathbb{R}^{N}$ da forma

$$
\left\{\begin{array}{cc}
-\Delta u+V(x) u=|u|^{p-1} u, & \text { em } \mathbb{m}^{N}, \\
u>0, & \text { em } \mathbb{R}^{N},
\end{array}\right.
$$

onde $N \geq 3 ; 1<p<\frac{N+2}{N^{r}-2}$ e o potencial $V: \mathbb{R}^{N} \rightarrow \mathbb{R}$ é de classe $C^{1}$ e limitado inferiormente.

A equação (1) surge no estudo de existência de ondas estacionárias para a equação de Schrödinger não linear do tipo

$$
i \hbar \frac{\partial \psi}{\partial t}=-\frac{\hbar^{2}}{2 m} \Delta \psi+V(x) \psi-\gamma|\psi|^{p-i} \psi
$$

Ou seja, quando se procura por soluções do problema (2) da forma $\psi(x, t)=e^{\frac{-i E t}{\hbar}} u(x)$, o problema se reduz a uma equação do tipo

$$
-h^{2} \Delta u-(V(x)-E) u=|u|^{p-1} u, \quad x \in \mathbb{R}^{N} .
$$

Uma escolha apropriada em $E$ torna $V-E$ limitado inferiomente por zero. Assim, sem perda de generalidade, podemos supor em todo o trabalho que $E=0$ e que $V$ é limitado inferiormente por uma constante positiva. Desta forma, a equação (3) pode ser escrita como

$$
-h^{2} \Delta u-V(x) u=|u|^{p-1} u, \quad x \in \mathbb{R}^{N}
$$

ou equivalentemente 


$$
-\Delta v-V(h x) v=|v|^{p-1} v, \quad x \in \mathbb{R}^{N},
$$

onde $v(x)=u(h x)$ e $\inf V>0$.

Em [6], Floer e Weinstein estudaram a equação (4) quando $N=1$ e $p=3$. Admitindo que o potencial $V$ é limitado estes autores provaram que para cada $h>0$ pequeno, existe uma solução $u_{h}$ de (4) cujo máximo global converge para cada ponto crítico não degenerado do potencial $V$, previamente fixado, o que caracteriza um fenômeno de concentração.

Em [12, 13], Oh generalizou esse resultado para dimensões superiores sob certas hipóteses no potencial $V$ no infinito e $1<p<\frac{N+2}{N-2}$.

Em [3], Ding e Ni provaram que se $N \geq 2$ e $V$ é radial então para todo $h>0$, a equação (4) possui uma solução radial positiva com energia mínima entre todas as soluções radiais não triviais em $H^{1}\left(\mathbb{R}^{N}\right)$.

Em [15], Rabinowitz provou que se o potencial $V$ for periódico, o problema (4) possui uma solução não trivial. Em [16], Rabinowitz mostrou que a equação (4) possui uma solução não trivial de energia mínima para todo $h>0$ se

$$
\liminf _{|x| \rightarrow \infty} V(x)=\sup _{x \in \mathbb{R}^{N}} V(x)
$$

ou para todo $h>0$ suficientemente pequeno se

$$
\liminf _{|x| \rightarrow \infty} V(x)>\inf _{x \in \mathbb{R}^{N}} V(x) .
$$

Em [17], Wang, motivado por uma questão feita por Rabinowitz em [16], estudou o comportamento de concentração das soluções positivas de energia mínima da equação (4) encontradas em [16], quando $h \rightarrow 0^{+}$. Wang provou que se (6) ocorre, então qualquer seqüência de soluções positivas de energia mínima da equação (4) contêm uma subseqüência concentrando em um ponto de mínimo global do potencial $V$, quando $h \rightarrow 0^{+}$.

O trabalho [4], de Felmer e del Pino, foi o primeiro a tratar o caso degenerado para a equação (4) do ponto de vista local. Eles criaram um método de penalização para encontrar soluções do tipo passo da montanha que se concentram em torno de mínimos locais 
de $V$, com degenerescência arbitrária. Em [5], Felmer e del Pino generalizaram o método para construir uma família de soluções com vários picos de concentração localizados nas proximidades de qualquer conjunto finito de mínimos locais de $V$ previamente fixado.

Muitos trabalhos tratam do caso em que o potencial $V$ é constante e a equação (4) é considerada em domínio limitado, entre os mais relevantes citamos os trabalhos de $\mathrm{Ni}$ e Takagi $[9,10]$, e Ni e Wei [11]. Em [9, 10], Ni e Takagi provaram que uma solução de energia mínima para o problema de Neumann

$$
\left\{\begin{aligned}
-h^{2} \Delta u+u-u^{p}=0, & & \text { em } \Omega, \\
u>0 \text { em } \Omega, \frac{\partial u}{\partial \eta}=0, & & \text { em } \partial \Omega,
\end{aligned}\right.
$$

possui exatamente um ponto de máximo local (portanto global) em $\bar{\Omega}$, isto é, um único pico, o qual localiza-se na fronteira de $\Omega$ e, a menos de subseqüência, concentra-se em torno de um ponto onde a curvatura média maximiza-se. Por outro lado, em [11], Ni e Wei provaram que uma solução de energia mínima do problema de Dirichlet

$$
\left\{\begin{array}{cc}
-h^{2} \Delta u+u-u^{p}=0, & \text { em } \Omega, \\
u>0 \text { em } \Omega, u=0, & \text { em } \partial \Omega,
\end{array}\right.
$$

necessariamente concentra-se em torno de um ponto mais central do domínio $\Omega$, isto é, em torno de um ponto de máxima distância a fronteira de $\Omega$.

Esta dissertação de Mestrado está organizada do seguinte modo: no Capítulo 1, seguindo Rabinowitz [15], estudamos o problema (1) para o caso em que o potencial $V$ é periódico. No Capítulo 2 , usando Rabinowitz [16], estudamos o mesmo problema quando potencial $V$ satisfaz a condição

$$
\liminf _{|x| \rightarrow \infty} V(x) \geq V_{\infty}
$$

para alguma constante positiva $V_{\infty}$.

Por fim, no Capítulo 3, explorando parte do artigo [17] de Wang estudamos o comportamento de concentração das soluções $u_{h}$ de (4) obtidas no Capítulo 2, quando $h \rightarrow 0$.

Informamos que os resultados abordados neste trabalho são válidos para uma classe mais geral de não linearidades $f(x, u)$. Preferimos lidar somente com o caso particular 
em que $f(x, u)=f(u)=u^{p}$, para evitar dificuldades técnicas adicionais e evidenciar os métodos utilizados para estudar a existência e a concentração de soluções. 


\section{Capítulo 1}

\section{Existência de Soluções para}

\section{Equações de Schrödinger com Coeficientes Periódicos}

Neste capítulo iremos considerar a seguinte equação:

$$
-\Delta u+V(x) u=|u|^{p-1} u, x \in \mathbb{R}^{N}
$$

satisfazendo:

(V) $V \in C^{1}\left(\mathbb{R}^{N}, \mathbb{R}\right)$ e é $X_{l}$ periódica em $x_{l}, 1 \leq l \leq N$.

$\left(V_{2}\right) \quad V(x)>0 \mathrm{em} \mathbb{R}^{N}$.

A dificuldade presente aqui é que em domínios ilimitados as imersões de Sobolev, em geral, não são compactas, de modo que o funcional associado a equação pode não satisfazer a condição de Palais-Smale. No entanto, sob a condição de periodicidade da função potencial $V$, o problema de existência de solução de (1.1) fica reduzido à análise do problema em domínio limitado.

A idéia, entāo, é mostrar que para cada $k \in \mathbb{N}$, existe uma solução $u_{k}$ de (1.1) que é $k X_{i}$ periódica em $x_{i}, 1 \leq i \leq N$. A existência de $u_{k}$ é dada por meio do Teorema do Passo da Montanha. Usando resultados de regularidade elíptica, obtemos uma estimativa uniforme de $u_{k}$, o que permite extrair uma subseqüência que converge para uma solução do problema (1.1). 
Teorema 1.1. Se (V $\left.V_{1}\right)-\left(V_{2}\right)$ são satisfeitas e $\left.1<p<\frac{N+2}{N-2}, N\right\rangle 2$, entäo a equação (1.1) possui uma solução clássica não trivial $u \in H^{1}\left(\mathbb{R}^{N}\right)$.

Observação 1.2. O resultado acima é válido para uma situação mais geral, mais precisamente quando a não linearidade $f(x, u)$ satisfaz:

$\left(f_{1}\right) f \in C^{1}\left(\mathbb{R}^{N} \times \mathbb{R}, \mathbb{R}\right)$ e é $X_{l}$ periódica ern $x_{l}, 1 \leq l \leq N$.

$\left(f_{2}\right) f(x, 0):=0=f_{u}(x, 0)$.

$\left(f_{3}\right)$ Existem constantes $b_{1}, b_{2}>0$ tais que

$$
|f(x, z)| \leq b_{1}+b_{2}|z|^{s}
$$

para todo $x \in \mathbb{R}^{N}, z \in \mathbb{R}$, onde $1<s<\frac{N+2}{N-2}$, se $N>2$.

$\left(f_{4}\right)$ Existe uma constante $\mu>2$ tal que

$$
0<\mu F(x, z) \equiv \mu \int_{0}^{z} f(x, t) d t \leq z(f(x, z),
$$

para todo $x \in \mathbb{R}^{N}, z \in \mathbb{R}-\{0\}$.

Vejamos a demonstração do Teorema 1.1.

Demonstraçāo: Seja

$$
Q_{k}=\left\{x \in \mathbb{R}^{N} \mid-\frac{k}{2} X_{i} \leq x_{i} \leq \frac{k}{2} X_{i}, 1 \leq i \leq N\right\}
$$

e considere o conjunto

$$
E_{k}=\left\{u \in H^{1}\left(Q_{k}\right): u \text { é } k X_{i} \text { periódico em } x_{i}, 1 \leq i \leq N\right\}
$$

O conjunto $E_{k}$ munido com a norma

$$
\|u\|_{k}^{2} \equiv \int_{Q_{k}}\left(|\nabla u|^{2}+V(x)|u|^{2}\right) d x
$$

é um espaço de Hilbert.

Para $u \in E_{k}$, considere o funcional

$$
I_{k}(u)=\frac{1}{2}\|u\|_{k}^{2}-\frac{1}{p+1} \int_{Q_{k}}|u|^{p+1} d x .
$$

Pelo Corolário A.6, Apêndice A, o funcional $I_{k} \in C^{2}\left(E_{k}, \mathbb{R}\right)$ e

$$
I_{k}^{\prime}(u) v=\int_{Q_{k}}(\nabla u \nabla v+V(x) u v) d x-\int_{Q_{k}}|u|^{p-1} u v d x
$$


para todo $v \in E_{k}$. Assim, os pontos críticos de $I_{k}$ em $E_{k}$ são solıções fracas de (1.1). Além dissó, tais soluções são $k X_{i}$ periódicas em $x_{i}, 1 \leq i \leq N$.

Para encontrar pontos críticos de $I_{k}$ em $E_{k}$, aplicamos o Teorema do Passo da Montanha de Ambrosetti-Rabinowitz [1], o qual faz uso da seguinte condição de compacidade.

Definição 1.3. Sejam $X$ um espaço de Banach, $I \in C^{1}(X, \mathbb{R})$. Uma seqüência $\left(u_{n}\right)$ é uma seqüência de Palais-Smale no nível c $\left((P S)_{c}\right)$ relacionada a I se

$$
I\left(u_{n}\right) \rightarrow c, \quad I^{\prime}\left(u_{n}\right) \rightarrow 0
$$

Dizemos que o funcional I satisfaz a condição $(P S)_{c}$ se toda seqüencia $(P S)_{c}$ possui uma subseqüência convergente em $X$.

Teorema 1.4. (Teorema do Passo da Montanha) Sejam $X$ um espaço de Hilbert e $I \in$ $C^{2}(X, \mathbb{R})$ satisfazendo $(P S)_{\mathrm{c}}$. Suponha que $I(0)=0$ e

$\left(\phi_{1}\right)$ Existem constantes $\rho, \alpha>0$ tais que $b:=\left.\inf I(u)\right|_{\partial B_{\rho}} \geq \alpha$,

$\left(\phi_{2}\right)$ Existe um $e \in X \backslash \overline{B_{\rho}}$ tal que $I(e) \leq I(0)$.

Entäo, I possui um valor crítico $c \geq \alpha$. Além disso, $c$ é caracterizado por

$$
c:=\inf _{\gamma \in \Gamma} \max _{t \in[0,1]} I(\gamma(t))
$$

onde

$$
\Gamma:=\{\gamma \in C([0,1], X): \gamma(0)=0, \gamma(1)=e\}
$$

Demonstração: Veja Teorema B.2, Apêndice B.

No que segue, com o objetivo de encontrar soluções fracas de (1.1), vamos verificar que $I_{k}$ satisfaz as condições geométricas $\left(\phi_{1}\right),\left(\phi_{2}\right)$ e a condição (PS).

Para verificar $\left(\phi_{2}\right)$, escolhendo $u \in E_{k}-\{0\}$ e $\beta \geq 0$ temos

$$
I_{k}(\beta u)=\frac{\beta^{2}}{2}\|u\|_{k}^{2}-\frac{\beta^{p+1}}{p+1} \int_{Q_{k}}|u|^{p+1} d x .
$$

Como $p+1>2, I_{k}(\beta u) \rightarrow-\infty$, quando $\beta \rightarrow \infty$. Portanto, $\left(\phi_{2}\right)$ é satisfeita.

Para verificar $\left(\phi_{1}\right)$, sendo $1<p<\frac{N+2}{N-2}$, temos pela imersão de Sobolev que existe uma constante $C>0$ tal que

$$
\|u\|_{p+1} \leq C\|u\|_{k}
$$


Assimi,

$$
I_{k}(u)=\frac{1}{2}\|u\|_{k}^{2}-\frac{1}{p+1} \int_{Q_{k}}|u|^{p+1} d x \geq \frac{1}{2}\|u\|_{k}^{2}-\frac{C}{p+1}\|u\|_{k}^{p+1}
$$

Portanto, para todo $u \in E_{k}$ tal que $|u|_{k}=\rho, \operatorname{com} \rho=\left(\frac{p+1}{\sharp C}\right)^{\frac{1}{\mu-1}}, \operatorname{temos} I_{k}(u) \geq \alpha$, $\alpha=\frac{\rho^{2}}{4} ;$ o que verifica a condição $\left(\phi_{1}\right)$.

A seguir, vamos verificar que $I_{k}$ satisfaz a condição de Palais-Smale $(P S)_{c}$ em $E_{k}$. Para isso, scja $\left(u_{m}\right)$ uma seqüencia em $E_{k}$ tal que

$$
I_{k}\left(u_{m}\right) \rightarrow c, \quad I_{k}^{\prime}\left(u_{m}\right) \rightarrow 0
$$

Primeiramente, vamos mostrar que (1.6) implica que a seqiiência $\left(u_{n n}\right)$ é limitada em $E_{k}$. De fato, fixe $2<\mu \leq p+1$, então

$$
\begin{aligned}
I_{k}\left(u_{m}\right)-\frac{1}{\mu} I_{k}^{\prime}\left(u_{m}\right) u_{m} & =\left(\frac{1}{2}-\frac{1}{\mu}\right)\left\|u_{m}\right\|_{k}^{2}+\left(\frac{1}{\mu}-\frac{1}{p+1}\right) \int_{Q_{k}}\left|u_{m}\right|^{p+1} d x \\
& \geq\left(\frac{1}{2}-\frac{1}{\mu}\right)\left\|u_{m}\right\|_{k}^{2} .
\end{aligned}
$$

Logo

$$
\frac{(\mu-2)}{2 \mu}\left\|u_{m}\right\|_{k}^{2} \leq\left|I_{k}\left(u_{m}\right)-\frac{1}{\mu} I_{k}^{\prime}\left(u_{m}\right) u_{m}\right| \leq \bar{M}+\frac{1}{\mu}\left\|I_{k}^{\prime}\left(u_{m}\right)\right\|\left\|u_{m}\right\|_{k}
$$

Suponhamos por contradição que $\left(u_{m}\right)$ não seja limitada em $E_{k}$. Logo, cxiste uma subseqüência $\left(u_{n_{i}}\right)$ de $\left(u_{m}\right)$ com $\left\|u_{n_{i}}\right\|_{k} \rightarrow \infty$. Dividindo ambos os lados de (1.7) por $\left\|u_{n_{i}}\right\|_{k}$, obternos

$$
\frac{(\mu-2)}{2 \mu}\left\|u_{n_{\mathrm{i}}}\right\|_{i k} \leq \frac{\bar{M}}{\left\|u_{n_{i}}\right\|_{k}}+\frac{1}{\mu}\left\|I_{k}^{\prime}\left(u_{n_{i}}\right)\right\| .
$$

Usando que $I_{k}^{\prime}\left(u_{n_{i}}\right) \rightarrow 0$, esta última expressão mostra que $\left(u_{n_{i}}\right)$ é limitada, contradizendo $\left\|u_{u_{i}}\right\|_{k} \rightarrow \infty$. Portanto, $\left(u_{m}\right)$ é limitada em $E_{k}$.

Tomando, se necessário, uma subseqüência de $\left(u_{m}\right)$, podemos admitir que

$$
\begin{aligned}
& u_{m} \rightarrow u \text { fraco em } E_{k} \\
& u_{m} \rightarrow u \text { forte em } L^{s}\left(Q_{k}\right), \text { para todo } 2 \leq s<2^{*}:=\frac{2 N}{N-2} .
\end{aligned}
$$

Em particular, para $s=p+1, u_{m} \rightarrow u$ em $L^{p+1}\left(Q_{k}\right)$. Agora, observe que

$$
\left\|u_{m}-u\right\|^{2}=I_{k}^{\prime}\left(u_{m}\right)\left(u_{m}-u\right)-I_{k}^{\prime}(u)\left(u_{m}-u\right)+\int_{Q_{k}}\left(\left(u_{m}\right)^{p}-(u)^{p}\right)\left(u_{m}-u\right)
$$


Combinando (1.6) e (1.9),

$$
I_{k}^{\prime}\left(u_{m}\right)\left(u_{m}-u\right)-I_{k}^{\prime}(u)\left(u_{m}-u\right) \rightarrow 0, \text { quando } m \rightarrow \infty
$$

Pela desigualdade de Hölder,

$$
\begin{aligned}
\left|\int_{Q_{k}}\left(\left(u_{m}\right)^{p}-(u)^{p}\right)\left(u_{m}-u\right) d x\right| & \leq C_{1}\left(\left\|u_{m}\right\|_{p+1}^{p}+\|u\|_{p+1}^{p}\right)\left\|u_{m}-u\right\|_{p+1} \\
& \leq C_{2}\left\|u_{m}-u\right\|_{p+1} \rightarrow 0, m \rightarrow \infty .
\end{aligned}
$$

Logo, de (1.10)-(1.12), $u_{m} \rightarrow u$ em $E_{k}$, quando $m \rightarrow \infty$, e com isto, $I_{k}$ satisfaz $(P S)_{c}$ em $E_{k}$.

Considere o nivel minimax associado ao funcional $I_{k}$ dado por

$$
c_{k}=\inf _{g \in \Gamma} \max _{\theta \in[0,1]} I_{k}(g(\theta))
$$

onde

$$
\Gamma_{k}=\left\{g \in C\left([0,1], E_{k}\right): g(0)=0, g(1)=e\right\}
$$

Pelo Tecrema do Passo da Montanha, $c_{k}$ é um valor crítico positivo de $I_{k}$, isto é, existe um $u_{k} \in E_{k}$ tal que

$$
I_{k}\left(u_{k}\right)=c_{k} \quad e \quad I_{k}^{\prime}\left(u_{k}\right)=0 .
$$

O que prova a existência de uma solução fraca não trivial de (1.1).

A condição em $p$ permite aplicar um argumento de regularidade conhecido na literatura por bootstrap para:

i) mostrar que $u_{k}$ é solução clássica da equação (1.1) e que pertence à $C^{2, a}\left(Q_{k}, \mathbb{R}\right)$ para todo $\alpha \in(0,1)$;

ii) fornecer estimativas para $u_{k}$ em algumas normas, principalmente em $L^{\infty}\left(Q_{k}\right)$ e $C^{2, \alpha}\left(Q_{k}, \mathbb{R}\right)$.

Observanıos que estas estimativas dependem de $k$. Assim, para mostrar que a seqüência $u_{k}$ converge para uma solução de (1.1), é necessário estabelecer uma estimativa uniforme de $u_{k}$ crn alguma dessas normas. Antes de fazermos isso, vamos estabelecer um limitante superior para $c_{k}$ que independa de $k$. 
Escolha $\varphi_{1} \in E_{1}-\{0\}$ tal que $\varphi_{1} \equiv 0$ numa vizinhança da $\partial Q_{1}$ e

$$
I_{1}\left(\varphi_{1}\right) \leq 0
$$

Defina $\gamma_{k}(x)=\varphi_{1}(x)$ em $Q_{1}$, e $\varphi_{k}(x)=0$ en $Q_{k} \backslash Q_{1}$. Estendendo $\varphi_{k}$ periodicamente. $\rho_{k} \in E_{k}$. Seja $g_{k}(\theta)=\theta \varphi_{k}$, para $\theta \in[0,1]$. Então, pela definição de $\varphi_{k}$ e por (1.16). temos que $g_{k} \in \Gamma_{k} \mathrm{e}$

$$
c_{k} \leq \max _{\theta \in[0,1]} I_{k}\left(g_{k}(\theta)\right)=\max _{\theta \in\{0,1]} I_{1}\left(\theta \varphi_{1}\right) \equiv M
$$

Note que $M$ independe de $k$.

A estimativa (1.17) fornece uma limitação superior para $u_{k}$ errı $E_{k}$ independente de $k$. Em verdade, temos:

$$
c_{k}=I_{k}\left(u_{k}\right)-\frac{1}{2} I_{k}^{i}\left(u_{k}\right) u_{k}=\left(\frac{1}{2}-\frac{1}{p+1}\right) \int_{Q_{k}}|u|^{p+1} d x
$$

Então por (1.4)-(1.5) e (1.17)-(1.18),

$$
\begin{aligned}
\left\|u_{k}\right\|_{k}^{2} & =2\left(c_{k}+\frac{1}{p+1} \int_{Q_{k}}|u|^{p+1} d x\right)=2\left(1+\frac{2}{p-1}\right) c_{k} \\
& \leq 2\left(1+\frac{2}{p-1}\right) M \equiv M_{1} .
\end{aligned}
$$

Proposição 1.5. Seja $O$ um conjunto aberto limitado em $\mathbb{R}^{N}$. Então, para qualquer $\alpha \in(0,1)$, existem constantes positivas $K_{1}$ e $K_{2}$ tais que

$$
\begin{gathered}
\left\|u_{k}\right\|_{L^{\infty}(O)} \leq K_{1} \\
\left\|u_{k}\right\|_{C^{2, \alpha}(O)} \leq K_{2}
\end{gathered}
$$

para todo $k \in \mathbb{N}$, com $K_{1}$ dependendo do diâmetro de $O$ e $K_{2}$ dependendo do diâmetro de O e de $\alpha$.

Demonstração: É sıficiente mostrar as estimativas para um $k$ suficientemente grande, em geral, $k \geq k_{0}$ onde $O \subset \subset Q_{k_{0}}$. Escolha $\hat{O}$ tal que $O \subset \subset \hat{O} \subset \subset Q_{k_{0}}$.

Pelas estimativas elípticas em $L^{p}$ (cf. Teorema 9.11, [8]), para todo $p \in(1, \infty)$ e $u \in W^{2, p}(\hat{O})$,

$$
\|u\|_{W^{2, p}(O)} \leq K_{3}\left(\|f(u)\|_{L^{p}(\hat{O})}+\|u\|_{L^{p}(\hat{O})}\right)
$$


Assim, como anteriomente: $\left(u_{k}\right)$ limitada em $L^{2^{*}}\left(O_{j}\right),\left(\left|u_{k}\right|^{p}\right)$ limitada em $L^{p_{0}}\left(O_{j}\right)$. implicava $\left(u_{k}\right)$ limitada em $W^{2, p_{0}}\left(O_{j-1}\right)$, agora temos: $\left(u_{k}\right)$ limitada em $L^{t_{0}}\left(O_{j}\right),\left(\left|u_{k}\right|^{p}\right)$ limitada em $L^{p_{1}}\left(O_{j}\right)$, o que implica $\left(u_{k}\right)$ limitada em $W^{2, p_{1}}\left(O_{j-1}\right)$, onde $p_{1}=\frac{t_{0}}{p}>\frac{2^{v}}{p}=p_{0}$.

Se $p_{1}>\frac{N}{2}$ então, aplicando o Passo $1,\left(u_{k}\right)$ é limitada em $L^{\infty}\left(O_{j-1}\right)$ e, portanto, $\left(u_{k}\right)$ é limitada em $L^{\infty}(O)$.

Se $p_{1}=\frac{N}{2}$, então, aplicando o Passo 2, $\left(u_{k}\right)$ é limitada em $L^{\infty}\left(O_{j \ldots 2}\right)$ e, portanto, $\left(u_{k}\right)$ é limitada $\mathrm{em} L^{\infty}(O)$.

Se $p_{1}<\frac{N}{2}$, então $(1.23)$ mostra que $\left(u_{k}\right)$ é limitada $e m L^{i_{1}}$ oilde.

$$
\frac{1}{t_{1}}=\frac{1}{p_{1}}-\frac{2}{N}=\frac{1}{p_{1}}-\frac{1}{p_{0}}+\frac{1}{p_{0}}-\frac{2}{N}=p\left(\frac{1}{t_{0}}-\frac{1}{2^{*}}\right)+\frac{1}{t_{0}} .
$$

Continuando este processo, na $j$-etapa teremos:

Oll $p_{j} \geq \frac{N}{2}$ e, portanto, $\left(u_{k}\right)$ é limitada em $L^{\infty}(O)$, ou $p_{j}<\frac{N}{2} \mathrm{e}$

$$
0<\frac{1}{t_{j}}=\frac{1}{p_{j}}-\frac{2}{N}=\sum_{i=1}^{j}\left(\frac{1}{p_{i}}-\frac{1}{p_{i-1}}\right)+\frac{1}{t_{0}}=p\left(\frac{1}{t_{0}}-\frac{1}{2^{*}}\right)+\frac{1}{t_{0}}
$$

Daí, $\quad 0<-j p\left(\frac{1}{2^{*}}-\frac{1}{t_{0}}\right)+\frac{1}{t_{0}}$, ou seja, $j p\left(\frac{1}{2^{*}}-\frac{1}{t_{0}}\right)<\frac{1}{t_{0}}$,

contrariando (1.26). Logo (1.20) está satisfeito.

Por fim, para provar (1.21) usaremos as estimativas de Schauder [8] para o problema (1.1). Para qualquer $\alpha \in(0,1)$ e $O \subset \subset \hat{O} \subset \subset Q_{k_{0}}$ :

$$
\left\|u_{k}\right\|_{C^{2, \alpha}(O)} \leq K_{5}\left(\left\|f\left(u_{k}\right)\right\|_{C^{\alpha}(\hat{O})}+\left\|u_{k}\right\|_{L^{\infty}(\hat{O})}\right)
$$

onde $f\left(u_{k}\right)=\left|u_{k}\right|^{p-1} u_{k}$ e $K_{5}$ depende de $N, \alpha$, dos diâmetros de $O$ e $(\hat{O}-O)$ e dà limitação $C^{\alpha}\left(Q_{1}\right)$ de $V$.

Por $(1.20)$ e $(1.27)$, para obter $(1.21)$, basta estimar $\left(f\left(u_{k}\right)\right)$ ern $C^{\complement c}(\hat{O})$. Para isso, usando a desigualdade do Valor Médio

$$
\frac{\left|f\left(u_{k}(x)\right)-f\left(u_{k}(y)\right)\right|}{|x-y|^{\alpha}} \leq \frac{\| D f\left(u_{k}(z)\right)|||| \nabla u_{k}(z)|||x-y|}{|x-y|^{\alpha}}
$$

$\operatorname{com} z=(1-t) x+t y$, para algum $t \in(0,1)$.

Agora, por (1.20) e usando (1.22) com $p>N$ obtemos uma limitação uniforme para $u_{k}$ em $C^{1}$, via imersão de Sobolev. Logo, existe uma constante positiva $K_{6}$ tal que, para todo $k$

$$
\left\|f\left(u_{k}\right)\right\|_{C^{\alpha}(\hat{O})} \leq K_{6}
$$


Consequentemente, por (1.27), a verificação de (1.21) está completa.

Observação 1.6. Combinando os resultados obtidos pelo processo de bootstrap temos:

$$
\left\|u_{k}\right\|_{L^{\infty}(O)} \leq K\left\|u_{k}\right\|_{H^{1}(\hat{O})} \leq K\left\|u_{k}\right\|_{H^{1}\left(\mathbb{P}^{N}\right)} \leq K M_{1}
$$

As limitações da Proposição 1.5 e o Teorema de Arzclà-Ascoli implicam que uma subseqüência de $\left(u_{k}\right)$ converge em $C_{l o c}^{2}\left(\mathbb{R}^{N}\right)$ para $u \in C_{l o c}^{2, \alpha}\left(\mathbb{R}^{N}\right)$ solução de (1.1). Além disso, por (1.19) temos que $u \in H^{1}\left(\mathbb{R}^{N}\right)$ e

$$
\|u\|_{H^{1}\left(\Omega^{N}\right)} \leq M_{1}
$$

Falta mostrar que $u$ é não trivial. Multiplicando a equação (1.1) por $u_{k}$ e integrando por partes obtemos

$$
\left\|u_{k}\right\|_{k}^{2}=\int_{Q_{k}}\left|u_{k}\right|^{p+1} d x
$$

Pela desigualdade de Poincaré

$$
\left\|u_{k}\right\|_{k}^{2} \leq K_{7}\left\|u_{k}\right\|_{L^{\infty}}^{p-1}\left\|u_{k}\right\|_{k}^{2}
$$

com $K_{7}$ independente de $k$. Como $\left\|u_{k}\right\|_{k}>0$ pois $I_{k}\left(u_{k}\right)=c_{k}>0$ temos,

$$
\left\|u_{k}\right\|_{L^{\infty}}^{p-1} \geq \frac{1}{K_{7}}>0 .
$$

Note que $I_{k}$ é simétrico em $\mathbb{Z}^{N}$, isto é, se $j=\left(j_{1}, \ldots, j_{N}\right) \in \mathbb{Z}^{N}, u \in E_{k}$ e

$$
\left(\tau_{j} u\right)(x)=u\left(x_{1}+j_{1} X_{1}, \ldots, x_{N}+j_{N} X_{N}\right)
$$

entäo

$$
I_{k}\left(\tau_{j} u\right)=I_{k}(u)
$$

Além disso, quando $u_{k}$ for ponto crítico de $I_{k}$ em $E_{k}$, então $\tau_{j} u_{k}$ também será para todo $j \in \mathbb{Z}^{N}$. Consequentemente,

$$
\max _{Q_{k}}\left|u_{k}\right|=\left\|u_{k}\right\|_{L^{\infty}\left(Q_{k}\right)}=\max _{Q_{1}}\left(\varphi_{k}\right)
$$

onde $\varphi_{k}$ é uma função construída a partír de $\tau_{j} u_{k}$, para algum $j \in \mathbb{Z}^{N}$, se necessário for (cf. página 5 a definição de $\varphi_{k}$ ).

Desde que $u_{k} \rightarrow u$ em $L_{l o c}^{\infty}$, se $u \equiv 0, u_{k} \rightarrow 0$ uniformente em $Q_{1}$, contrariando (1.30). Logo $u$ é uma solução não trivial da equação (1.1). 


\section{Capítulo 2}

\section{Existência de Soluções para}

\section{Equações de Schrödinger}

Neste capítulo, consideraremos o seguinte problema:

$$
\begin{cases}-\Delta u+V(x) u=u^{p}, & \mathrm{em} \mathbb{R}^{N} \\ u>0, & \mathrm{em} \mathbb{R}^{N}\end{cases}
$$

no qual, $1<p<\frac{N+2}{N-2}$, se $N \geq 3$ ou $1<p$ se $N=2$ e $V \in C^{1}\left(\mathbb{R}^{N}\right)$ satisfaz:

$\left(V_{3}\right)$ Existe $V_{0}>0$ tal que $V(x) \geq V_{0}$ para todo $x \in \mathbb{R}^{N}$.

$\left(V_{1}\right)$ Existe uma constante positiva $V_{\infty}$ tal que

$$
\liminf _{|x| \rightarrow \infty} V(x) \geq V_{\infty}
$$

A prova da existência de solução para (2.1) será feita usando métodos variacionais. Para isto, consideraremos $E$ o subespaço de $H^{1}\left(\mathbb{R}^{N}\right)$ munido da seguinte norma:

$$
\|u\|^{2} \equiv \int_{\mathbb{R}^{N}}\left(|\nabla u|^{2}+V(x)|u|^{2}\right) d x
$$

Por $\left(V_{3}\right), E$ está imerso continuamente em $H^{1}\left(\mathbb{R}^{N}\right)$. Definimos o funcional $I: E \rightarrow \mathbb{R}$ associado ao problema (2.1) e dado por

$$
I(u)=\frac{1}{2} \int_{\mathbb{R}^{N}}\left(|\nabla u|^{2}+V(x) u^{2}\right) d x-\frac{1}{p+1} \int_{\mathbb{R}^{N}}\left(u^{+}\right)^{p+1} d x
$$

onde $u^{+}=\max \{u, 0\}$. 
Pelo Corolário A.6, Apêndice A, o funcional $I$ está bem definido em $E$ e $I \in C^{2}(E, \mathbb{R})$. Além disso, todo ponto crítico de $I$ é uma solução fraca de (2.1). Aplicando os argumentos utilizados no Capítulo 1, essa solução fraca também é uma solução clássica de (2.1).

Uma condição necessária para que $u \in E$ seja um ponto crítico de $I$ é que

$$
I^{\prime}(u) u=0
$$

Esta condição define o que na literatıra é chamado de variedade de Nehari e dada por

$$
\mathcal{N}=\left\{u \in E-\{0\}: \int_{\mathbb{R}^{N}}\left(|\nabla u|^{2}+V(x) u^{2}\right) d x=\int_{\mathbb{E}^{N}}\left(u^{+}\right)^{p+1} d x\right\}
$$

Tal qual fizemos no Capítulo 1, o funcional $I$ satisfaz as hipóteses do Teorema do Passo da Montanha, exceto possivelmente a condição Palais-Smale. No entanto, utilizando propriedades dos níveis de energia, mostraremos que $I$ possui um valor crítico positivo. A seguir recordaremos a definição de seqüência $(P S)_{c}$ e enunciaremos a versão do Teorema do Passo da Montanha que utilizaremos:

Definição 2.1. Sejam $X$ um espaço de Bunach, $I \in C^{1}(X, \mathbb{R})$. Uma seqüência $\left(u_{n}\right)$ é uma seqüência de Palais-Smale no nível c (seqüencia $(P S)_{c}$ ) relacionada a I se

$$
I\left(u_{n}\right) \rightarrow c, \quad I^{\prime}\left(u_{n}\right) \rightarrow 0
$$

Teorema 2.2. Sejam $X$ um espaço de Hilbert real e $I \in C^{2}(X, \mathbb{R})$. Suponha que $I(0)=0$ e

$\left(\phi_{1}\right)$ Existem constantes $\rho, \alpha>0$ tais que $b:=\left.\inf I(u)\right|_{\partial B_{\rho}} \geq \alpha$,

$\left(\phi_{2}\right)$ Existe um $e \in X \backslash \overline{B_{\rho}}$ tal que $I(e) \leq I(0)$.

Então, existe uma seqüencia $(P S)_{c}, \operatorname{com} c \geq \alpha$, onde

$$
c:=\inf _{g \in \Gamma} \max _{t \in[0,1]} I(g(t))
$$

sendo

$$
\Gamma:=\{g \in C([0,1], X): g(0)=0, g(1)=e\}
$$

Demonstração: Veja Teorema B.2 do Apêndice B.

No que segue daremos uma caracterização do nível minimax c obtido no Teorema anterior. Primeiramente notemos que para qualquer $u \in E-\{0\}$ e $t>0$, definindo

$$
\sigma(t) \equiv I(t u)=\frac{t^{2}}{2} \int_{\mathbb{R}^{N}}\left(|\nabla u|^{2}+V(x) u^{2}\right) d x-\frac{t^{p+1}}{p+1} \int_{\mathbb{R}^{N}}\left(u^{+}\right)^{p+1} d x
$$


temos

i) $\sigma(0)=0$,

ii) $\sigma(t)<0$ para $t$ suficientemente grande,

iii) $\sigma(t)>0$ para $t$ suficientemente pequeno.

Por isso, o $\max _{t \geq 0} \sigma(t)$ existe e como veremos é atingido para um único valor de $t$. De fato, $\sigma^{\prime}(t)=0$ é equivalente a

$$
t\|u\|^{2}-t^{p} \int\left(u^{+}\right)^{p+1} d x=0
$$

o que implica.

$$
t=\left(\frac{\|u\|^{2}}{\|u\|_{p+1}^{p+1}}\right)^{\frac{1}{p-1}}
$$

pois $u \in E-\{0\}$.

Consequentemente, isto permite definir uma aplicação $\varphi(u): E-\{0\} \rightarrow \mathbb{R}^{+}$cue a cada $u \in E-\{0\}$ faz corresponder $t=\varphi(u)$ para o qual

$$
\max _{t \geq 0} \sigma(t)=\sigma(\varphi(u))
$$

Além disso, para cada $u \in E-\{0\}, t=\varphi(u)$ é o único valor de $t>0$ tal que tu $\in \mathcal{N}$. Para ver isto, basta multiplicar (2.5) por $t=\varphi(u)$.

Proposição 2.3. A aplicação $E-\{0\} \rightarrow \mathbb{R}^{+}: u \mapsto \varphi(u)$ é contínua.

Demonstraçäo: Seja $u_{m} \rightarrow u$ em $E-\{0\}$. Então

$$
\varphi\left(u_{m}\right)^{2}\left\|u_{m}\right\|^{2}=\int_{\mathbb{R}^{N}}\left(\varphi\left(u_{m}\right) u_{m}^{+}\right)^{p+1} d x
$$

Temos que $\varphi\left(u_{m}\right) \leq 1$ ou $\varphi\left(u_{m}\right)>1$. Como veremos a seguir basta considerarmos este último caso. Seja $\mu \in(2, p+1)$, então

$$
\begin{aligned}
\int_{\mathbb{R}^{N}}\left(\varphi\left(u_{m}\right) u_{m}^{+}\right)^{p+1} d x & \geq \frac{\mu}{p+1} \int_{\mathbb{R}^{N}}\left(\varphi\left(u_{m}\right) u_{m}^{+}\right)^{p+1} d x \\
& \geq \frac{\mu}{p+1} \int_{\mathbb{R}^{N}}\left[\varphi\left(u_{m}\right)\right]^{\mu}\left(u_{m}^{+}\right)^{p+1} d x .
\end{aligned}
$$

Consequentemente

$$
\varphi\left(u_{m}\right)^{2}\left\|u_{m}\right\|^{2}=\int_{\mathbb{R}^{N}} \varphi\left(u_{m}\right)^{p+1}\left(u_{m}^{+}\right)^{p+1} d x \geq \frac{\mu}{p+1} \varphi\left(u_{m}\right)^{\mu} \int_{\mathbb{R}^{N}}\left(u_{m}^{+}\right)^{p+1} d x
$$


o que implica que

$$
\varphi\left(u_{m}\right)^{\mu-2} \leq \frac{(p+1)\left\|u_{m}\right\|^{2}}{\mu \int_{\mathbb{R}^{N}}\left(u_{m}^{+}\right)^{p+1} d x} \rightarrow \frac{(p+1)\|u\|^{2}}{\mu \int_{\mathbb{R}^{*}}\left(u^{+}\right)^{p+1} d x}, \text { quando } m \rightarrow \infty .
$$

Segne que $\varphi\left(u_{m}\right)$ é limitada, e uma subseqüência de $p\left(u_{m}\right)$ converge para $\bar{\varphi}$.

Se $\bar{\varphi}=0$, dividimos (2.6) por $\varphi\left(u_{m}\right)^{2}$ e obtemos $\|u\|=0$ quando $m \rightarrow \infty$, contrariando assim que $u \in E-\{0\}$.

Logo $\bar{\varphi}>0$ e passando o limite em (2.6) obtemos:

$$
\bar{\varphi}^{2}\|u\|^{2}=\int_{\mathbb{R}^{N}} \vec{\vartheta}^{p+1}\left(u^{+}\right)^{p+1} d x
$$

o que implica que $\bar{\varphi} u \in \mathcal{N}$. Pela unicidade de $\varphi(u), \bar{\varphi}=\varphi(u)$. Segue então que

$$
\varphi\left(u_{m}\right) \rightarrow \bar{\varphi}=\varphi(u)
$$

Agora defina

$$
c^{*} \equiv \inf _{\substack{u \in E \\ u \neq 0}} \max _{\theta \geq 0} I(\theta u)
$$

Logo obtemos o seguinte resultado:

Proposição 2.4. $c^{*}=c=\inf _{\mathcal{N}} I$.

Demonstração: Para cada $u \in E-\{0\}$ temos que

$$
\max _{\theta \geq 0} I(\theta u)=I(\varphi(u) u) \quad \text { e } \quad \varphi(u) u \in \mathcal{N}
$$

segue então que $c^{*}=\inf _{\mathcal{N}} I$.

Seja $g \in \Gamma$. Afirmamos que $g([0,1]) \cap \mathcal{N} \neq \emptyset$. De fato, se $u \in E-\{0\}$ pertence à $\mathcal{N}$

$$
\|u\|^{2}=\int_{\mathbb{R}^{N}}\left(u^{+}\right)^{p+1} d x \geq \frac{\mu}{p+1} \int_{\mathbb{R}^{N}}\left(u^{+}\right)^{p+1} d x
$$

para todo $\mu \in(2, p+1)$ fixado, logo

$$
I(u)=\frac{1}{2}\|u\|^{2}-\frac{1}{p+1} \int_{\mathbb{R}^{N}}\left(u^{+}\right)^{p+1} d x \geq\left(\frac{\mu}{2}-1\right) \int_{\mathbb{R}^{N}} \frac{\left(u^{+}\right)^{p+1}}{p+1} d x>0 .
$$

Desde que $g(0)=0$ e $I(g(1))<0$ segue que $g([0,1]) \cap \mathcal{N} \neq \emptyset$. Então

$$
\max _{t \in[0,1]} I(g(t)) \geq \min _{u \in \mathcal{N}} I(u)=c^{*}
$$


$\log 0, \geq c^{*}$

Por outro lado, para $u \in E-\{0\}$ dado, tem-se $I(\theta u)<0$ para $\theta$ suficientemente grande.

Na verdade, cada raio $\theta u: \theta \geq 0$ pode ser associado com a função $g_{u} \in \mathrm{I}$, tomando o devido reescalonamento em $\theta$ para que $g_{u}(1)<0$.

Consequentemente

$$
c^{*}=\inf _{u \in E} \max _{\theta \geq 0} I(\theta u)=\inf _{u \in E} \max _{t \in\left[0, L_{j}^{:}\right.} I\left(g_{u}(t)\right) \geq \inf _{g \in \Gamma \in[0,1]} \max _{t \in[(g(t))=c}
$$

o que implica que $c^{*} \geq c$. Portanto, $c=c^{*}$.

Observação 2.5. Como $c=\inf _{\mathcal{N}} I$ e todo ponto crítico de I pertence a $\mathcal{N}$, se c é valor crítico positivo de I, ele é o menor valor crítico positivo de I.

Mostraremos a seguir que o nível minimax $c$ depende continuamente da função potencial $V$.

Lema 2.6. Sejam $V$ e $\widehat{V}$ satisfazendo $\left(V_{3}\right)$ com os valores minimax c e $\widehat{c}$ correspondentes. Se $V \geq \widehat{V}$ então $c \geq \widehat{c}$.

Demonstração: Se $V \geq \widehat{V}$, então se $\widehat{I}$ é o funcional associado com $\widehat{V}$ temos $I(u) \geq \widehat{I}(u)$, para todo $u \in E$. Seja $\widehat{\Gamma}$ o análogo a $\Gamma$ para $\widehat{I}$., então, $g \in \Gamma$ implica $g \in \widehat{\Gamma}(\Gamma \subset \widehat{\Gamma})$, logo

$$
\max _{t \in[0,1]} I(g(t)) \geq \max _{t \in[0,1]} \widehat{I}(g(t))
$$

Consequentemente

$$
c \geq \inf _{g \in \bar{\Gamma}} \max _{t \in[0,1]} \widehat{I}(g(t)) \geq \inf _{g \in \widehat{\Gamma}} \max _{t \in[0,1]} \widehat{I}(g(t))=\widehat{c} .
$$

Teorema 2.7. Sejam $V, V_{m}$ satisfazendo $\left(V_{3}\right)$, para todo $m \in \mathbb{N}$. Se $V_{m} \rightarrow V$ em $L^{\infty}\left(\mathbb{R}^{N}\right)$ então $c\left(V_{m}\right) \rightarrow c(V)$, isto é, c depende continuamente de $V$.

Demonstração: Sejà $\epsilon>0$, então para $m$ suficientemente grande

$$
V+\epsilon \geq V+\left|V_{m}-V\right| \geq V \geq V-\left|V_{m}-V\right| \geq V-\epsilon
$$


Com isto c pelo Lema 2.6 é suficiente mostrar um resultado mais simples:

$$
c_{\epsilon} \equiv c(V+\epsilon) \rightarrow c_{0}, \text { quando } \epsilon \rightarrow 0
$$

Iremos mostrar primeiro para $\epsilon<0$. Pelo Lema 2.6

$$
\lim _{\varepsilon \rightarrow 0^{-}} c_{\epsilon}=c \leq c_{0}
$$

Suponha que $\underline{c}<c_{0}$.

$$
I_{\epsilon}(u)=\int_{\mathbb{R}^{N}}\left[\frac{1}{2}\left(|\nabla u|^{2}+(V+\epsilon) u^{2}\right)-\frac{\left(u^{+}\right)^{p+1}}{p+1}\right] d x .
$$

Seja $\epsilon_{k} \rightarrow 0^{-}$, quando $k \rightarrow \infty$ e $\delta_{m} \rightarrow 0^{+}$quando $m \rightarrow \infty$. Para cada $k$, pela Proposição 2.4, sem perda de generalidade podemos supor que existe uma seqüencia $\left(u_{k m}\right) \subset E$ tal que $\left\|u_{k \pi n}\right\|=1 \mathrm{e}$

$$
\max _{\theta \geq 0} I_{\epsilon_{k}}\left(\theta u_{k m}\right) \leq c_{\epsilon_{k}}+\delta_{m}
$$

Assim, como na demonstração da Proposição 2.4, para cada $u_{k m}$ associamos a finnção $g_{u_{k, n}} \in \Gamma_{k}$ tal que

$$
\max _{t \in[0,1]} I_{\epsilon_{k}}\left(g_{u_{k m}}(t)\right)=\max _{\theta \geq 0} I\left(\theta u_{k m}\right)
$$

Pelo Corolário C.3, Apêndice C, existem seqüências $\left(w_{k m}\right) \subset E$ e $\left(t_{k m}\right) \subset[0,1]$ tizis que

$$
\begin{gathered}
\left\|w_{k m}-g_{u_{k m}}\left(t_{k m}\right)\right\| \leq \delta_{m}^{1 / 2} \\
I_{\epsilon_{k}}\left(w_{k m}\right) \in\left(c_{c_{k}}-\delta_{m}, c_{\epsilon_{k}}\right), \\
\left\|I_{\epsilon_{k}}^{\prime}\left(w_{k m}\right)\right\|_{1} \leq \delta_{m}^{1 / 2} .
\end{gathered}
$$

Tomarıdo $m=k$, seja $u_{k} \equiv u_{k k}$, então:

$$
\begin{aligned}
c_{0} & \leq \max _{\theta \geq 0} I\left(\theta u_{k}\right)=I\left(\varphi\left(u_{k}\right) u_{k}\right) \\
& =I_{\epsilon_{k}}\left(\varphi\left(u_{k}\right) u_{k}\right)-\epsilon_{k} \varphi\left(u_{k}\right)^{2} \int_{\mathbb{R}^{N}} u_{k}^{2} d x \\
& \leq \max _{\theta \geq 0} I_{\epsilon_{k}}\left(\theta u_{k}\right)-\epsilon_{k} \varphi\left(u_{k}\right)^{2} \int_{\mathbb{R}^{N}} u_{k}^{2} d x \\
& \leq \epsilon_{\epsilon_{k}}+\delta_{k}-\epsilon_{k} \varphi\left(u_{k}\right)^{2} \int_{\mathbb{R}^{N}} u_{k}^{2} d x \\
& \leq \underline{c}+\delta_{k}-\epsilon_{k} \varphi\left(u_{k}\right)^{2} \int_{\mathbb{R}^{N}} u_{k}^{2} d x
\end{aligned}
$$

onde a última desigualdade é consequência do Lema 2.6 . 
Como $\left\|u_{k}\right\|=1$, existe uma constante $M_{1}$ tal que

$$
\left\|u_{k}\right\|_{L^{2}\left(\Omega^{*}\right)} \leq M_{1}
$$

Portanto, se $\left(\varphi\left(u_{k}\right)\right)$ é limitada e com limitação independente de $k$, basta tomarnos $\epsilon_{\dot{k}}$ suficientemente pequeno para que (2.9) contrarie a hipótese $\left(\underline{c}<c_{0}\right)$.

Afirmamos que $\varphi\left(u_{k}\right)$ é limitada. De fato. se, ao longo de uma subseqüência, $\varphi\left(u_{k}\right) \leq 1$ nada a demonstrar. Se $\varphi\left(u_{k}\right)>1$ então fazendo análogo a demonstração da Proposição 2.3 teremos

$$
\varphi\left(u_{k}\right)^{\mu-2} \leq \frac{(p+1)}{\mu \int_{\mathbb{R}^{v}}\left(u_{k}\right)^{p+1} d x} .
$$

Se, ao longo de uma subseqüência, o termo $\left(u_{k}\right)^{p+1}$ for limitado e distante de zero, teremos um limitante superior para $\varphi\left(u_{k}\right)$. Caso contrário, quando $k \rightarrow \infty$

$$
\int_{\mathbb{R}^{N}}\left(u_{k}\right)^{p+1} d x \rightarrow 0
$$

Usando (2.8) mostraremos que (2.10) não ocorre. A aplicação $g_{u_{k}}(t) \equiv g_{u_{k k}}(t)$ tem a forma $\psi_{k}(t) u_{k}$. Além disso, $g_{u_{k}}\left(t_{k}\right)=\psi_{k}\left(t_{k}\right) u_{k} \equiv \xi_{k} u_{k}$ e por $(2.8)$,

$$
\left\|w_{k}-\xi_{k} u_{k}\right\| \leq \delta_{k}^{1 / 2}
$$

Assim como feito na demonstração do Teorema 1.1, (2.8) implica que $\left(w_{n_{2}}\right)$ é limitado em $E$. Além disso, de $(2.11)$ e $\left\|u_{k}\right\|=1$, existe uma constante $M_{2}$ independente de $k$ tal que

$$
\xi_{k} \leq \delta_{k}^{1 / 2}+\left\|w_{k}\right\| \leq M_{2}
$$

Por (2.12), para qualquer $r>0$ e $y \in \mathbb{R}^{N}$,

$$
\begin{aligned}
\left\|u_{k}\right\|_{L^{2}\left(B_{r}(y)\right)} & \geq M_{2}^{-1}\left\|\xi_{k} u_{k}\right\|_{L^{2}\left(B_{r}(y)\right)} \\
& \geq M_{2}^{-1}\left(\left\|w_{k}\right\|_{L^{2}\left(B_{r}(y)\right)}-\left\|w_{k}-\xi_{k} u_{k}\right\|_{L^{2}\left(B_{r}(y)\right)}\right) \\
& \geq M_{2}^{-1}\left(\left\|w_{k}\right\|_{L^{2}\left(B_{r}(y)\right)}-M_{3} \delta_{k}^{1 / 2}\right)
\end{aligned}
$$

onde $M_{3}$ é constante.

Afirmação: Existe uma seqüência $\left(y_{k}\right)$ em $\mathbb{R}^{N}$ e constantes $R>0$ e $\beta>0$ tais que

$$
\liminf _{k \rightarrow \infty} \int_{B_{R}\left(y_{k}\right)} w_{k}^{2}(x) d x \geq \beta>0 .
$$


De fato, suponhamos por contradição que a afirmação seja falsa. Encão, para torlo $R>0$ dado,

$$
\lim _{k \rightarrow \infty} \sup _{y \in \mathbb{R} . N} \int_{B_{R}(y)} w_{k}^{2}(x) d x=0
$$

Consequentemente, pelo Lema de Lions (cf. Lema D.1, Apêndice D)

$$
u_{k} \rightarrow 0 \operatorname{em~} L^{q}\left(\mathbb{R}^{N}\right)
$$

para todo $2<q<\frac{2 N}{N-2}$. Mas as limitaçōes de $\left\|w_{k}\right\|$ e (2.8) implicam que

$$
I_{\epsilon_{k}}\left(u_{k}\right)-\frac{1}{2} I_{\epsilon_{k}}^{\prime}\left(w_{k}\right) w_{k} \rightarrow \underline{c}>0 .
$$

Por outro lado, por (2.15) temos

$$
I_{\epsilon_{k}}\left(w_{k}\right)-\frac{1}{2} I_{\epsilon_{k}}^{\prime}\left(w_{k}\right) w_{k}=\int_{\mathbb{R}^{v}}\left(\frac{1}{2}-\frac{1}{p+1}\right) w_{m}^{p+1} d x \rightarrow 0
$$

contrariando (2.16), verificando assim (2.14). Fazendo $y=y_{k}$ e $r=R$ em (2.13) temos para $k$ suficientemente grande,

$$
\left\|u_{k}\right\|_{L^{2}\left(B_{R}\left(y_{i}\right)\right)} \geq M_{2}^{-1}\left(\frac{\beta}{2}\right)^{1 / 2}
$$

Agora, para provar que (2.10) não ocorre é suficiente mostrar que existe um $\beta_{1}>0$ tal que

$$
\int_{B_{R}\left(y_{k}\right)}\left(u_{k}\right)^{p+1} d x \geq \beta_{1}
$$

Mas, usando a desigualdade de Hölder, existe uma constante positiva $C$ que depende de $N$ e $R$ tal que

$$
\int_{B_{R}\left(y_{k}\right)}\left(u_{k}\right)^{p+1} d x \geq C\left\|u_{k}\right\|_{L^{2}\left(B_{R}\left(y_{k}\right)\right)}^{p+1} \geq C\left[M_{2}^{-1}\left(\frac{\beta}{2}\right)^{1 / 2}\right]^{p+1} \doteq \beta_{1} .
$$

Isto completa a demonstração de $(2 . \overline{7})$ para o caso $\epsilon<0$. Argumentos similares são utilizados para provar (2.7) para o caso $\epsilon>0$. No que segue faremos um roteiro dos passos da demonstração neste caso. Pelo Lema 2.6,

$$
c_{0} \leq \bar{c}=\lim _{\epsilon \rightarrow 0^{+}} c_{\epsilon}
$$

Suponha que $c_{0}<\bar{c}$. Seja $\delta_{k}$ como introduzido anteriormente. Pela Proposição 2.4, existe uma seqüência $\left(u_{k}\right) \subset E$ tal que $\left\|u_{k}\right\|=1 \mathrm{e}$

$$
\max _{\theta \geq 0} I\left(\theta u_{k}\right)=c_{0}+\delta_{k}
$$


Escolha $w_{k}=w_{k k}$ como em (2.8). Para cada $\epsilon \geq 0$ e $u \in E-\{0\}$ considere $\varphi_{\epsilon}(u)$ scndo para $I_{\epsilon}$ o que $\varphi(u)$ é para $I$. Então por (2.18),

$$
\begin{aligned}
\bar{c} & <c_{c} \leq \max _{\theta \geq 0} I_{\epsilon}\left(\theta u_{k}\right)=I_{\epsilon}\left(\varphi_{\epsilon}\left(u_{k}\right) u_{k}\right) \\
& =I\left(\varphi_{\epsilon}\left(u_{k}\right) u_{k}\right)+\epsilon \varphi_{\epsilon}\left(u_{k}\right)^{2} \int_{\mathbb{R}^{N}} u_{k}^{2} d x \\
& \leq c_{0}+\delta_{k}+\epsilon \varphi_{i}\left(u_{k}\right)^{2} \int_{\mathbb{R}^{N}} u_{k}^{2} d x .
\end{aligned}
$$

Se $\varphi_{\varepsilon}$ é limitada então (2.19) contraria a hipótese $\left(c_{0}<\bar{c}\right)$ e (2.7) está prorado.

Como feito anteriormente, ou $\varphi_{\epsilon} \leq 1$ ou

$$
\varphi_{\epsilon}\left(u_{k}\right)^{\mu-2} \leq \frac{(p+1) \int_{\mathbb{R}^{N}}\left[\left|\nabla u_{k}\right|^{2}+\left((V(x)+\epsilon) u_{k}\right)^{2}\right] d x}{\mu \int_{\mathbb{R}^{N}}\left(u_{k}\right)^{p+1} d x} .
$$

Os argumentos utilizados para o caso $\epsilon<0$ e o fato de $\left\|u_{k}\right\|=1$ mostram que $\varphi_{\epsilon}\left(u_{k}\right)$ ć limitada para $\epsilon$ suficientemente pequeno, o que completa a demonstração do Teorema 2.7 .

Enunciaremos a seguir um resultado crucial para provar a existência de solução do problema (2.1), cuja prova é baseada em argumentos de comparação entre os níveis minimax. Varnos primeiramente introduzir algumas notações. Denotemos por $c_{\infty}$ o nível minimax associado ao funcional

$$
I_{\infty}(u)=\int_{\mathbb{R}^{N}} \frac{1}{2}\left(|\nabla u|^{2}+V_{\infty} u^{2}\right) d x-\frac{1}{p+1} \int_{\mathbb{R}^{N}}\left(u^{\top}\right)^{p+1} d x
$$

definido em $E$, com $V_{\infty}$ dado por $\left(V_{4}\right)$.

Teorema 2.8. Suponha que $V$ satisfaça $\left(V_{3}\right)$ e $\left(V_{4}\right)$. Então c é um valor crítico de $I$ ov. $c \geq c_{\infty}$

Demonstração: Primeiramente suponhamos que $V$ satisfaça uma versão mais forte da condição $\left(V_{4}\right)$, a saber,

$$
\liminf _{|x| \rightarrow \infty} V(x)>V_{\infty}
$$

Pela caracterização de $c$ dada na Proposição 2.4 , existe uma seqüiência $\left(u_{m}\right) \subset E$ tal que $\left\|u_{m}\right\|=1 \mathrm{e}$

$$
\max _{\theta \geq 0} I\left(\theta u_{m}\right) \rightarrow c, \text { quando } m \rightarrow \infty
$$


Assim como feito na Proposição 2.4 , a cada $u_{m}$ associa-se uma função $g_{n} \in \Gamma$ tal que

$$
\max _{t \in[0,1]} I\left(g_{m}(t)\right)=\max _{\theta \geq 0} I\left(\theta u_{m}\right)
$$

Polo Corolário C.3, do Apêndice C, existem seqüências $\left(w_{m}\right) \subset E, 0<\epsilon_{m} \rightarrow 0$, c $t_{m} \in[0,1]$ tais que

$$
\begin{gathered}
\left\|w_{m}-g_{m}\left(t_{m}\right)\right\| \leq \epsilon_{m}^{1 / 2}, \\
I\left(w_{m}\right) \in\left(c-\epsilon_{m}, c\right), \\
\left\|I^{\prime}\left(w_{m}\right)\right\| \leq \epsilon_{m}^{1 / 2}
\end{gathered}
$$

Assim como feito na demonstração do Teorema 1.1, (2.23) implica que $\left(w_{m}\right)$ é limitada em $E$. Além disso, uma subseqüência de $w_{m}$ converge fracamente em $E$ e fortemente em $L_{l o c}^{p}\left(\mathbb{R}^{N}\right), 1 \leq p<2^{*}$, para uma funçāo $w$, solução fraca de (2.1).

Afirmação: Existe uma seqüência $\left(y_{m}\right)$ em $\mathbb{R}^{N}$ e constantes $R>0$ e $\beta>0$ tais que

$$
\liminf _{m \rightarrow 0^{+}} \int_{B_{R}\left(y_{m}\right)} w_{m}^{2}(x) d x \geq \beta>0 .
$$

De fato, se (2.24) não é válido então

$$
\lim _{m \rightarrow 0^{+}} \sup _{y \in \mathbb{R}^{N}} \int_{B_{R}(y)} w_{m}^{2}(x) d x=0 .
$$

Pelo Lema D.1, $w_{m} \rightarrow 0$ em $L^{q}\left(\mathbb{R}^{N}\right)$, para qualquer que seja $2<q<2^{*}$. Em particular, $u_{n} \rightarrow 0$ em $L^{p+1}\left(\mathbb{R}^{N}\right)$. Por outro lado,

$$
I\left(w_{m}\right)-\frac{1}{2} I^{\prime}\left(w_{m}\right) w_{m}=\left(\frac{1}{2}-\frac{1}{p+1}\right) \int_{\mathbb{B}^{N}}\left|w_{m}\right|^{p+1} d x
$$

Como o lado direito da equação (2.25) tende a zero quando $m \rightarrow \infty$, encontramos uma contradição com (2.23), verificando assim a afirmação.

Se $\left(y_{m}\right)$ contém uma subseqüência limitada, por $(2.24)$ podemos admitir $w \neq 0$. Além disso, para qualquer $r>0$ temos,

$$
I\left(w_{m}\right)-\frac{1}{2} I^{\prime}\left(w_{m}\right) w_{m}=\left(\frac{1}{2}-\frac{1}{p+1}\right) \int_{\mathbb{R}^{N}}\left|u_{m}\right|^{p+1} d x \geq\left(\frac{1}{2}-\frac{1}{p+1}\right) \int_{B_{r}(0)}\left|w_{m}\right|^{p+1} d x
$$

Por (2.23) e pelas imersões de Sobolev segue que

$$
c \geq\left(\frac{1}{2}-\frac{1}{p+1}\right) \int_{\mathbb{R}^{N}} \mid w^{p+1} d x
$$


Mas $w \in E$ é uma solução clássica de (2.1), consequentemente o lado direito da equação (2.26) é igual a $I(u)$. Logo, segue da Observação 2.5 e $w \neq 0$ quc $I(u)=c$, concluindo assim a demonstração do Teorema 2.8 para este caso.

Suponhamos agora que $\left(y_{m}\right)$ seja ilimitada. Fixemos $V_{0}<\mu<V_{\infty}$ e consideremos $r>0$ tal que

$$
V(x) \geq \mu, \quad \forall x \in \mathbb{R}^{N} ;|x| \geq r
$$

Entäo, pará todo $t>0$,

$$
\begin{aligned}
\max _{\theta \geq 0} I\left(\theta u_{m}\right) & \geq I\left(t u_{m}\right)=I_{\mu}\left(t u_{m}\right)+\frac{1}{2} \int_{\mathbb{R}^{N}}(V(x)-\mu)\left|t u_{m}\right|^{2} d x \\
& =\frac{1}{2} \int_{B_{r}(0)}(V(x)-\mu)\left|t u_{m}\right|^{2} d x+\frac{1}{2} \int_{\mathbb{R}^{N} \backslash B_{r}(0)}(V(x)-\mu)\left|t u_{m}\right|^{2},
\end{aligned}
$$

onde

$$
I_{\mu}(u)=\frac{1}{2} \int_{\mathbb{R}^{N}}\left(|\nabla u|^{2}+\mu u^{2}\right) d x-\frac{1}{p+1} \int_{\mathbb{R}^{N}}|u|^{p+1} d x
$$

Por $(2.27)$,

$$
\max _{\theta \geq 0} I\left(\theta u_{m}\right) \geq I_{\mu}\left(t u_{m}\right)+\frac{1}{2} \int_{B_{r}(0)}(V(x)-\mu)\left|t u_{m}\right|^{2} d x, \quad \forall t \geq 0 .
$$

Escolhendo $t=\varphi_{\mu}\left(u_{m}\right)$, onde $\varphi_{\mu}\left(u_{m}\right)$ é dado por (2.5) para $I_{\mu}$, obtemos

$$
I_{\mu}\left(\varphi_{\mu}\left(u_{m}\right)\right)=\max _{\theta \geq 0} I_{\mu}\left(\theta u_{m}\right)
$$

Então, pela Proposiçāo 2.4 para $I_{\mu}$, e por $(2.29)$

$$
\max _{\theta \geq 0} I\left(\theta u_{m}\right) \geq c_{\mu}+\frac{1}{2} \int_{B_{r}(0)}(V(x)-\mu)\left(\varphi_{\mu}\left(u_{m}\right) u_{m}\right)^{2} d x
$$

Como na demonstração do Teorema 2.7 , a seqüência $\left(\varphi_{\mu}\left(u_{m}\right)\right)$ é limitada.

Suponhamos que exista um $\gamma_{1}>0$ tal que

$$
\left\|u_{m}\right\|_{L^{2}\left(B_{r}(0)\right)} \geq \gamma_{1}
$$

Assim, como na demonstração do Teorema $2.7, g_{u_{n n}}\left(t_{m n}\right) \equiv \xi_{m} u_{m}$ e por $(2.23)$,

$$
\left\|\xi_{m} u_{m}-w_{m}\right\| \leq \epsilon_{k}^{1 / 2}
$$

Logo

$$
\left\|w_{m}\right\|_{L^{2}\left(B_{r}(0)\right)} \geq\left\|\xi_{m} u_{m}\right\|_{L^{2}\left(B_{r}(0)\right)}-\left\|w_{m}-\xi_{m} u_{m}\right\|_{L^{2}\left(B_{r}(0)\right)}
$$


Por (2.33), o termo de $w_{m}$ do lado direito de (2.34) tende a zero quando $m \rightarrow \infty$. So $\xi_{n} \rightarrow 0$ ao longo de uma subseqüència, $\xi_{m} u_{m} \rightarrow 0$ e $I\left(\xi_{m} u_{m}\right) \rightarrow 0$, o que contraria (2.22). Portanto, $\left(\xi_{m}\right)$ tem um limitante inferior positivo e (2.34) mostra que cxiste um $\gamma_{2}>0$ tal que

$$
\left\|u_{m}\right\|_{L^{2}\left(B_{r}(0)\right)} \geq \gamma_{2}
$$

Análogo ao caso em cue $\left(y_{m}\right)$ limitado, $u_{m}$ converge fracamente em $E$ para uma solução $u$ de $(2.1) \operatorname{com} I(u)=c$.

Se (2.32) não ocorre, então tomando uma subseqüência de $\left(u_{m}\right)$, ainda denotada por $\left(u_{n}\right)$, temos

$$
\left\|u_{m}\right\|_{L^{2}\left(B_{r}(0)\right)} \rightarrow 0, \quad m \rightarrow \infty
$$

Por (2.22), (2.31), (2.35) e pela limitação de $\left(\varphi_{\mu}\left(u_{m}\right)\right)$ temos

$$
c \geq c_{\mu} .
$$

Fazendo agora $\mu \rightarrow V_{\infty}$ e usando o Teorema 2.7 obtemos

$$
c \geq c_{\infty}
$$

Portanto, a demonstração do Teorema 2.8 está completa para o caso em que (2.21) é válida.

Suponhamos agora que $\liminf _{|x| \rightarrow \infty} V(x)=V_{\infty}$. Então para todo $\epsilon>0$,

$$
\liminf _{|x| \rightarrow \infty} V(x)>V_{\infty}-\epsilon .
$$

Logo, pelos resultados já provados, ou $(i) c$ é um valor crítico de $I$ ou $(i i) c \geq c_{\infty}(\epsilon)$, onde $c_{\infty}(\epsilon)$ é o nível minimax associado ao funcional

$$
I_{\epsilon}(u)=\int_{\mathbb{R}^{N}} \frac{1}{2}\left(|\nabla u|^{2}+\left(V_{\infty}-\epsilon\right) u^{2}\right) d x-\frac{1}{p+1} \int_{\mathbb{R}^{N}}\left(u^{+}\right)^{p+1} d x .
$$

Suponhamos que $(i)$ não seja válido. Então fazendo $\epsilon \rightarrow 0$ e usando o Teorema 2.7 obtemos $c \geq c_{\infty}$, concluindo assim a demonstração do Teorema 2.8 .

Corolário 2.9. Se $1<p<\frac{N+2}{N-2}$ quando $N \geq 3$ ou $p>1$ quando $N=1,2$ então $c_{\infty}$ é um valor crítico de $I_{\infty}$ com uma correspondente solução clássica $u_{\infty}$ do problema:

$$
-\Delta u+V_{\infty} u=|u|^{p-1} u, x \in \mathbb{R}^{N} .
$$


Demonstração: Pelo Corolário C.3, do Apêndice C, existe uma seqüência $\left(w_{m}\right) \subset E$, tal que

$$
\begin{gathered}
I_{\infty}\left(w_{m}\right) \rightarrow c_{\infty}, \\
I_{\infty}^{\prime}\left(w_{m}\right) \rightarrow 0 .
\end{gathered}
$$

Como visto anteriormente, as funções $w_{m}$ são limitadas em $E$ e existem uma seqüência $\left(y_{m}\right) \subset \mathbb{R}^{N}, \beta \in R>0$ tais que

$$
\liminf _{m \rightarrow 0^{+}} \int_{B_{R}\left(y_{m}\right)} u_{m}^{2}(x) d x \geq \beta>0
$$

Observe que se $y \in \mathbb{R}^{N}, u \in E$ e se $\left(\tau_{y} u\right)(x) \equiv u(x-y)$ então $I_{\infty}\left(\tau_{y} u\right)=I_{\infty}(u)$. Na verdade, transladando $w_{m}$ se necessário, podemos admitir

$$
\liminf _{m \rightarrow 0^{+}} \int_{B_{R}(0)} u_{m}^{2}(x) d x \geq \beta>0
$$

e os argumentos da demonstração do Teorema 2.8 implicam que $w_{m}$ converge fracamente em $E$ para uma soluçāo $u_{\infty}$ de $(2.37)$, com $I_{\infty}\left(u_{\infty}\right)=c_{\infty}$.

Observação 2.10. Combinando o Teorema 2.8 com o Lema 2.6, notemos que para qualquer $\nu \in\left[V_{0}, V_{\infty}\right]$ o valor minimax correspondente $c_{\nu}$ é um valor crítico do funcional associado $I_{\nu}$.

No resultado que segue, mostraremos que quando $V$ satisfaz una condição mais forte que $\left(V_{4}\right)$, o valor minimax $c$ é um valor crítico.

Teorema 2.11. Suponhamos que $V \in C^{1}\left(\mathbb{R}^{N}\right)$ satisfaça $\left(V_{3}\right)$ e

$\left(V_{5}\right) \quad \liminf _{|x| \rightarrow \infty} V(x) \equiv V_{\infty} \geq V(x), \quad \forall x \in \mathbb{R}^{N}, \quad \operatorname{com} V_{\infty} \neq V(x)$.

Então cé um valor crítico de $I$.

Demonstração: Se c não ć um valor crítico de $I$ então, pelo Teorema $2.8, c \geq c_{\infty}$. Seja $w$ um ponto crítico de $I_{\infty}$ correspondente ao nível $c_{\infty}$ então

$$
c_{\infty}=I_{\infty}(w)=\max _{\theta \geq 0} I_{\infty}(\theta w)
$$

Para qualquer $\theta>0$,

$$
I_{\infty}(\theta w)=I(\theta w)+\frac{1}{2} \int_{\mathbf{R}^{N}}\left(V_{\infty}-V\right)|\theta w|^{2} d x
$$


Tomando $\theta=\varphi(w)$ obtemos por $\left(V_{5}\right),(2.38)$ e $(2.39)$

$$
\begin{aligned}
c_{\infty} & \geq I(\varphi(w) w)+\frac{1}{2} \int_{\Xi^{*}}\left(V_{\infty}-V\right)|\varphi(w) w|^{2} d x \\
& \geq c+\frac{1}{2} \int_{\mathbb{R}^{N}}\left(V_{\infty}-V\right)|\varphi(w) w|^{2} d x>c
\end{aligned}
$$

contrariando, assim, o Teorema 2.8. Portanto, $c$ é valor crítico de $I$.

O resultado final deste capítulo estabelece a existência de solução para o seguinte problema singularmente perturbado:

$$
\left\{\begin{array}{cc}
-h^{2} \Delta u+V(x) u=u^{p}, & \text { em } \mathbb{R}^{N} \\
u>0, & \text { em } \mathbb{R}^{N}
\end{array}\right.
$$

onde $h>0$ é um parâmetro real, $1<p<\frac{N+2}{N-2}$ se $N \geq 3$ ou $1<p$ se $N=1,2$ e o potencial $V$ satisfaz a condição $\left(V_{3}\right)$.

Notemos que via mudança de variáveis $x \mapsto h x$ em $\mathbb{R}^{N}$ o problema (2.41) é equivalente a

$$
\left\{\begin{array}{cc}
-\Delta v+V(h x) v=v^{p}, & \text { em } \mathbb{R}^{N} \\
v>0, & \text { em } \mathbb{R}^{N}
\end{array}\right.
$$

no qual $v(x)=u(h x)$.

Teorema 2.12. Suponhamos que $V$ satisfaça $\left(V_{3}\right)$ e

$$
\liminf _{|x| \rightarrow \infty} V(x) \geq V_{\infty}>\inf _{x \in \mathbb{R}^{N}} V(x)
$$

Então existe um $h_{0}>0$ tal que (2.42) possui uma solução clássica para todo $h \in\left(0, h_{0}\right)$.

Demonstração: Sem perda de generalidade, vamos admitir que $V_{0}=V(0)=\inf _{x \in \mathbb{R}^{N}} V(x)$.

Seja $h>0$ e considere $\widetilde{E}$ o subespaço de $H^{1}\left(\mathbb{R}^{N}\right)$ munido da seguinte norma:

$$
\|u\|^{2} \equiv \int_{\mathbb{R}^{N}}\left(|\nabla u|^{2}+V(h x)|u|^{2}\right) d x
$$

Como $\inf _{\mathbb{R}^{N}} V>0, \tilde{E}$ está imerso continuamente em $H^{1}\left(\mathbb{R}^{N}\right)$. Definimos o funcional $I_{h}$ : $\widetilde{E} \rightarrow \mathbb{R}$ associado ao problema $(2.42)$ e dado por

$$
I_{h}(u)=\frac{1}{2} \int_{\mathbb{R}^{N}}\left(|\nabla u|^{2}+V(h x) u^{2}\right) d x-\frac{1}{p+1} \int_{\mathbb{R}^{N}}\left(u^{+}\right)^{p+1} d x .
$$


Pelo Corolário A.6, Apêndice A, o funcional $I_{h}$ está bem definido em $\widetilde{E}$ e $I_{h} \in C^{2}(\widetilde{E}, \mathbb{R})$. Denotemos por $c_{h}$ o valor minimax do funcional $I_{k}$ e dado por $(2.4)$.

Fixemos $V_{0}<\nu<V_{\infty}$. Pelo Lema 2.6,

$$
c_{p}<c_{\infty}
$$

onde $c_{\nu}$ é o nível minimax do funcionaì $I_{\nu}$ dado por

$$
I_{\nu}(u)=\frac{1}{2} \int_{\mathbb{R}^{N}}\left(|\nabla u|^{2}+\nu u^{2}\right) d x-\frac{1}{p+1} \int_{\mathbb{R}^{N}}|u|^{p+1} d x .
$$

Seja $w \in H^{1}\left(\mathbb{R}^{N}\right), w>0$, tal que

$$
I_{\nu}(w)=c_{\nu} \text { e } I_{\nu}^{\prime}(w)=0
$$

A existência de $u$ é dada pela Observaçāo 2.10 .

Para cada $R>0$, seja $w_{R}(x)=\eta_{R}(x) w(x)$, onde $\eta_{R} \in C^{\infty}\left(\mathbb{R}^{N}\right), 0 \leq \eta \leq 1, \eta_{R} \equiv 1$ em $B_{R}(0), \eta_{R} \equiv 0$ em $\mathbb{R}^{N} \backslash B_{2 R}(0)$ e $\left|\nabla \eta_{R}\right| \leq C(N)$. Pelo Teorema da Convergência Dominada de Lebesgue

$$
w_{R} \rightarrow w \text { em } \tilde{E} \text {, quando } R \rightarrow+\infty
$$

De (2.44) e pela Proposição $2.3, \varphi\left(w_{R}\right) \rightarrow \varphi(w)=1$, quando $R \rightarrow+\infty$. Assim,

$$
\varphi\left(w_{R}\right) w_{R} \rightarrow w \operatorname{em} \widetilde{E}, \text { quando } R \rightarrow+\infty
$$

Consequentemente

$$
I_{\nu}\left(\varphi\left(w_{R}\right) w_{R}\right)=I_{\nu}(w)+\left(I_{\nu}\left(\varphi\left(w_{R}\right) w_{R}\right)-I_{\nu}(w)\right)=I_{\nu}(w)+o_{R}(1)
$$

onde $o_{R}(1) \rightarrow 0$ : quando $R \rightarrow \infty$.

Consideremos $R>0$ suficientemente grande de modo que

$$
I_{\nu}\left(\varphi\left(u_{R}\right) w_{R}\right)<c_{\infty}
$$

Seja agora

$$
\begin{aligned}
I_{h}\left(t w_{R}\right) & =I_{\nu}\left(t w_{R}\right)+\frac{t^{2}}{2} \int_{R^{N}}(V(h x)-\nu) w_{R}^{2} d x \\
& =I_{\nu}\left(t w_{R}\right)+\frac{t^{2}}{2} \int_{B_{2 R}(0)}(V(h x)-\nu) w_{R}^{2} d x
\end{aligned}
$$


Consideremos $0<h<1$. Pela desigualdade do Valor Médio, existe uma constante $C(R) \geq 0$ tal que

$$
|V(h x)-V(0)| \leq C(R) h
$$

onde $C(R)=2 R\|\nabla V\|_{L^{\infty}\left(\bar{B}_{2 R}(0)\right)}$.

Usando $V(0)=V_{0}$ e $V_{0}<\nu$, segue de $(2.4 \overline{7})$ que

$$
V(h x)-\nu<V_{0}-\nu+C(R) h
$$

$\log 0$

$$
V(h x)-\nu<0, \quad \forall h \in\left(0, \frac{\nu-V_{0}}{C(R)}\right) .
$$

Observamos que $C(R)$ poderia ser nula se $V$ fosse constante em $B_{2 R}(0)$. Mas, nesse caso, a condição (2.48) ocorreria para todo $h \in(0,1)$.

Defirindo $h_{0}=\operatorname{minin}\left\{1, \frac{\nu-V_{0}}{C(R)}\right\}$, segue de $(2.46)$ e (2.48) que

$$
I_{h}\left(t w_{R}\right)<I_{\nu}\left(t w_{R}\right), \quad \forall h \in\left(0, h_{0}\right), t>0 .
$$

$\log O$

$$
\max _{t>0} I_{h}\left(t w_{R}\right) \leq \max _{t>0} I_{\nu}\left(t w_{R}\right)=I_{\nu}\left(\varphi\left(w_{R}\right) w_{R}\right)
$$

Assim, por (2.45) e (2.49),

$$
c_{h}=\inf _{w \in \tilde{E}-\{0\}} \max _{t>0} I_{h}(t w) \leq I_{\nu}\left(\varphi\left(w_{R}\right) w_{R}\right)<c_{\infty}
$$

desde que $h \in\left(0, h_{0}\right)$. Usando o Teorema $2.8, c_{h}$ é um valor crítico de $I_{h}$ e, consequentemente, o problema (2.42) possui uma solução clássica.

Observação 2.13. Em [16] é provado que os resultados anteriores são válidos para uma situação mais geral, mais precisamente quando a não linearidade $f(x, u)$ satisfaz:

$\left(f_{1}\right) f \in C^{2}\left(\mathbb{R}^{N} \times \mathbb{R}, \mathbb{R}\right)$,

$\left(f_{2}\right) f(x, 0)=0=f_{z}(x, 0)$.

$\left(f_{3}\right)$ Existem constantes $c_{1}, c_{2}>0$ e $1<s<2^{*}$ tais que para todo $x \in \mathbb{R}^{N}$ e $z \in \mathbb{R}$

$$
\left|f_{z}(x, z)\right| \leq c_{1}+c_{2}|z|^{s-1}
$$


$\left(f_{4}\right)$ Existe uma constante $\mu>2$ tal que

$$
0<\mu F(x, z) \equiv \mu \int_{0}^{z} f(x, t) d t \leq z(f(x, z))
$$

para todo $x \in \mathbb{R}^{N}: z \in \mathbb{R}-\{0\}$.

$\left(f_{5}\right) t^{-1} z f(x, t z)$ ć função crescente em $t>0$ para todo $x \in \mathbb{R}^{N}$ e $z \in \mathbb{E}-\{0\}$. 


\section{Capítulo 3}

\section{Concentração de Soluções de}

\section{Equações de Schrödinger}

Neste capítulo, seguindo os argumentos de Wang [17], mostraremos que para todo $h>0$ suficientemente pequeno, os máximos globais $x_{h}$ das soluções $u_{h}$ encontradas no Capítulo 2 concentram-se em torno de um mínimo global da função potenciail $V$, quando $h \rightarrow 0$.

Considere o seguinte problema:

$$
\left\{\begin{array}{cc}
-h^{2} \Delta u+V(x) u=u^{p}, & \text { em } \mathbb{R}^{N}, \\
u>0, & \text { em } \mathbb{R}^{N},
\end{array}\right.
$$

ou equivalentemente

$$
\left\{\begin{array}{cc}
-\Delta v+V(h x) v=v^{p}, & \text { em } \mathbb{R}^{N} \\
v>0, & \text { em } \mathbb{R}^{N}
\end{array}\right.
$$

no qual $v(x)=u(h x), 1<p<\frac{N+2}{N-2}$ se $N \geq 3$ ou $1<p$ se $N=1,2$ e o potencial $V$ satisfaz a condição:

$$
\liminf _{|x| \rightarrow \infty} V(x)>V_{0} \equiv \inf _{\mathbb{B}^{N}} V>0
$$

Mais precisamente, provaremos o seguinte resultado.

Teorema 3.1. Suponhamos que $V$ satisfaça $\left(V_{7}\right)$. Então existe $h_{0}>0$ tal que para todo $0<h<h_{0}$ existe uma solução $u_{h}$ de (3.1). Além disso, existe uma seqüêricia $h_{k} \rightarrow 0$ tal 
que $u_{h_{k}} \equiv u_{k}$ possui exatamente um máximo local (portanto, global) $x_{k} \in \mathbb{R}^{N}$. Também, $V\left(x_{k}\right) \rightarrow \inf _{x \in \mathbb{R}^{*}} V(x)$, quando $k \rightarrow \infty, e$

$$
u_{k}(x) \leq C \exp \left(-\frac{M}{h}\left|x-x_{k}\right|\right)
$$

para certas constantes $C, M$.

Observação 3.2. A demonstração do Teoremc 3.1 é longa. Primeiramente prova-se que

$$
w_{h}(\cdot)=u_{h}\left(h \cdot+x_{h}\right) \rightarrow u_{0}(\cdot), \text { em } C_{\text {loc }}^{2}\left(\mathbb{R}^{N}\right), L^{\infty}\left(\mathbb{R}^{N}\right) \text { e } H^{2}\left(\mathbb{R}^{N}\right), \text { quando } h \rightarrow 0^{+},
$$

onde un é a única solução do problema limite

$$
\left\{\begin{array}{l}
-\Delta u+V_{0} u=u^{p}, \quad e m \mathbb{R}^{N}, \\
u>0, e m \mathbb{R}^{N}, \\
u(0)=\max _{x \in \mathbb{S}^{N}} u(x) \\
u(x) \rightarrow 0,|x| \rightarrow \infty .
\end{array}\right.
$$

A idéia central da demonstração do Teorema 3.1 é comparar a solução $w_{n}$ do nível minimax corn a solução $u_{0}$ do problema limite (3.3). Por Gidas-Ni-Nirenberg [7], $u_{0}$ é radial, $u_{0}^{\prime}(r)<0$ para todo $r \neq 0$, e

$$
u_{0}(r),\left|u_{0}^{\prime}(r)\right| \leq C r^{(N-1) / 2} e^{-\sqrt{V_{0}^{r}}}
$$

para uma constante positiva $C$.

Em vista do Teorema 2.12, para demonstrarmos o Teorema 3.1, basta estudarmos o comportamento de concentração da família de soluções $v_{h}(x)=u_{h}(h x)$ de (3.2) obtidas no Capítulo 2. Para tanto necessitamos de alguns resultados dados pelos lemas que so seguem.

Lema 3.3. Seja $c_{0}$ o valor minimax do Passo da Montanha do funcional $I_{0}$ dado por

$$
I_{0}(u)=\frac{1}{2} \int_{\Omega}\left(|\nabla u|^{2}+V_{0} u^{2}\right) d x-\frac{1}{p+1} \int_{\Omega}\left(u^{+}\right)^{p+1} d x
$$

Então,

$$
\lim _{h \rightarrow 0} c_{h}=c_{0} \text {. }
$$


Demonstração: Pela Observação 2.10 do Capítulo 2 e a caracterização do nivel minimax, o problema

$$
\left\{\begin{array}{cc}
-\Delta w+V_{0} w=w^{p}, & \text { em } \mathbb{R}^{N} \\
w>0 & \mathrm{em} \mathbb{R}^{N}
\end{array}\right.
$$

possui uma solução de menor energia $w \in H^{1}\left(\mathbb{R}^{N}\right)$, isto é, $w$ satisfaz

$$
c_{0}=I_{0}(w)=\inf _{v \in I^{1}} \sup _{\theta \geq 0} I_{0}(\theta v) .
$$

Para cada $r>0$ seja $w_{r}(x)=\eta_{r}(x) w(x)$, onde $\eta_{r}$ é uma função corte com suporte compacto na bola $B_{2 r}(0), \eta_{r} \equiv 1$ em $B_{r}(0)$ e $\left|\nabla \eta_{r}\right| \leq C(N)$. Desse modo, pelo Teorema da Convergência Dominada de Lebesgue

$$
w_{r} \rightarrow \text { wem } H^{1}\left(\mathbb{R}^{N}\right) \text {, quando } r \rightarrow+\infty
$$

Agora, para cada $h$ e $r$ positivos, existe $t_{h, r}>0$ tal que

$$
I_{h}\left(t_{h, r} w_{r}\right)=\max _{t \geq 0} I_{h}\left(t w_{r}\right)
$$

Portanto,

$$
t_{h, r}^{p-1} \int_{B_{2 r}(0)} w_{\tau}^{p+1} d x=\int_{B_{2 r}(0)}\left(\left|\nabla w_{r}\right|^{2}+V(h x) w_{r}^{2}\right) d x
$$

Para cada $r>0$ fixado, a família $\left\{t_{h, r}\right\}_{h}$ é limitada. De fato, de (3.6) temos que

$$
t_{h, r}^{p-1} \int_{B_{2 r}(0)} w_{r}^{p+1} d x=\int_{B_{2 r}(0)}\left(\left|\nabla w_{r}\right|^{2}+V_{0} w_{r}^{2}\right) d x+\int_{B_{2 r}(0)}\left(V(h x)-V_{0}\right) w_{r}^{2} d x
$$

Pelo Teorema da Convergência Dominada de Lebesgue

$$
\lim _{h \rightarrow 0} \int_{B_{2 r}(0)}\left(V(h x)-V_{0}\right) u_{r}^{2} d x=0
$$

Assim, de (3.7) e (3.8), segue que $\left\{t_{h, r}\right\}_{h}$ limitada. Sem perda de generalidade, podemos supor que existe $t_{r} \geq 0$ tal que $t_{h, r} \rightarrow t_{r}$, quando $h \rightarrow 0$. Assim, fazendo $h \rightarrow 0 \mathrm{em}$ (3.7), obtemos

$$
t_{r}^{p-1} \int_{B_{2 r}(0)} w_{r}^{p+1} d x=\int_{B_{2 r}(0)}\left(\left|\nabla w_{r}\right|^{2}+V_{0} u_{r}^{2}\right) d x
$$


De (3.5) e (3.9),

$$
\begin{aligned}
t_{r}^{p-1} \int_{\mathbb{R}^{N}}\left|w_{r}\right|^{p+1} d x & =\int_{\mathbb{R}^{N}}\left(\left|\nabla u_{\mathrm{r}}\right|^{2}+V_{0} w_{r}^{2}\right) d x \\
& =\int_{\mathbb{R}^{N}}\left(\mid \nabla w_{i}^{2}+V_{0} w^{2}\right) d x+o_{r}(1) \\
& =\int_{\mathbb{R}^{N}}|u|^{p+1} d x+o_{r}(1)=\int_{\mathbb{R}^{N}}\left|w_{r}\right|^{p+1} d x+o_{r}(1)
\end{aligned}
$$

onde $o_{r}(1) \rightarrow 0$ quando $r \rightarrow \infty$.

Daí,

$$
\left(t_{r}^{p-1}-1\right) \int_{\mathbb{R}^{N}}\left|w_{r}\right|^{p+1} d x=o_{r}(1)
$$

Por outro lado, novamente por $(3.5), w_{r} \rightarrow w$ em $L^{p+1}\left(\mathbb{R}^{N}\right)$, com $\|w\|_{p+i}^{p+1} \neq 0$ pois

$$
\|w\|_{p+1}^{p+1}=\|w\|_{2}^{2} \neq 0
$$

Portanto, fazendo $r \rightarrow \infty$ em (3.10), obtemos $t_{r} \rightarrow 1$.

Defina $g(t)=I_{0}\left(t u_{r}\right)$ em $[0, \infty)$. Sob a condição em $p$ e por (3.9), concluimos que o máximo de $g$ em $[0, \infty)$ é atingido somente em $t_{r}$, isto é,

$$
I_{0}\left(t_{r} w_{r}\right)=\max _{t \geq 0} I_{0}\left(t w_{r}\right)
$$

Pela caracterizaçāo de $c_{h}$,

$$
c_{h} \leq \max _{i \geq 0} I_{h}\left(t w_{r}\right)=I_{h}\left(t_{h, r} w_{r}\right)
$$

o que nos leva à segninte desigualdade

$$
\limsup _{h \rightarrow 0^{+}} c_{h} \leq I_{0}\left(t_{r} w_{r}\right)
$$

Agora, de (3.5) e da convergência de $\left(t_{r}\right)$, obtemos

$$
\limsup _{h \rightarrow 0^{+}} c_{h} \leq \lim _{r \rightarrow \infty} I_{0}\left(t_{r} w_{r}\right)=I_{0}(w)=c_{0}
$$

Por outro lado, sendo $V(h z) \geq V_{0}$ para todo $z \in \mathbb{R}^{N}$, segue que $c_{h} \geq c_{0}$ para todo $h$. Logo

$$
\liminf _{h \rightarrow 0^{+}} c_{h} \geq c_{0}
$$

O que nos permite concluir que $c_{h} \rightarrow c_{0}$, quando $h \rightarrow 0^{+}$. 
Observemos agora que, como

$$
\left(\frac{1}{2}-\frac{1}{p+1}\right) \int_{\mathbb{R}^{N}}\left(\left|\nabla v_{h}\right|^{2}+V(h x) v_{h}^{2}\right) d x=I_{h}\left(v_{h}\right)-\frac{1}{p+1} I_{h}^{\prime}\left(v_{h}\right) v_{h}=c_{h},
$$

usando o Lema 3.3 , segue que $\left(v_{h}\right)$ é limitada.

Lema 3.4. Existe uma familia $\left\{y_{h}\right\}$ em $\mathbb{R}^{N}$ e constantes positivas $R$ e $\beta$ tais que

$$
\liminf _{h \rightarrow 0^{+}} \int_{B_{R}\left(y_{h}\right)} v_{h}^{2}(x) d x \geq \beta>0
$$

Demonstração: Suponhamos, por contradição, que o lema seja falso. Então, para todo $R>0$ dado, existe uma seqüência $v_{n}=v_{h_{n}}$ tal que

$$
\lim _{n \rightarrow \infty} \sup _{y \in \mathbb{R}^{N}} \int_{B_{R}(y)} v_{n}^{2}(x) d x=0, \quad \forall R>0 .
$$

Pelo Lema de Lions (cf. Lema D.1, Apêndice D), $v_{n} \rightarrow 0$ em $L^{q}\left(\mathbb{R}^{N}\right)$, para todo $2<q<$ $\frac{2 N}{N-2}$. Por outro lado, $v_{n}$ verifica

$$
\left.\int_{\mathbb{R}^{N}}\left(\left|\nabla v_{n}\right|^{2}+V_{h_{n}}(z)\right) v_{n}^{2}\right) d z=\int_{\mathbb{R}^{N}} v_{n}^{p+1} d z
$$

$\operatorname{logo}$, teríamos $v_{n} \rightarrow 0$ em $H^{1}\left(\mathbb{R}^{N}\right)$ e, conseqüentemente, $c_{h_{n}} \rightarrow 0$; o que é um absurdo, pois, pelo Lema $3.3, c_{h_{n}} \rightarrow c_{0}>0$.

Lema 3.5. A familia $\left\{h y_{h}\right\}_{h}$ é limitada para todo $h>0$ pequeno.

Demonstração: Suponhamos, por contradição, que exista uma seqüencia $\left(h_{n}\right)$ tal que $h_{n} y_{h_{n}} \rightarrow \infty$. Consideremos a seqüência

$$
w_{n}(x)=v_{n}\left(x+y_{n}\right)
$$

onde $y_{n}=y_{h_{n}}$ e estudemos seu comportamento quando $n \rightarrow \infty$. A função $w_{n}$ é solução clássica de

$$
-\Delta w_{n}+V\left(h_{n} x+h_{n} y_{n}\right) w_{n}=w_{n}^{p}, \quad w_{n}>0 \mathrm{em} \mathbb{R}^{N}
$$

Pelo Lema 3.4,

$$
\liminf _{n_{i} \rightarrow \infty} \int_{B_{R}(0)} w_{n}^{2}(x) d x \geq \beta>0
$$


Pela limitação de $v_{h}$, a função $w_{n}$ é limitada em $H^{1}\left(\mathbb{R}^{N}\right)$ e, portanto, a menos de subseqüencia, podemos admitir que $w_{n} \rightarrow w_{0} \geq 0$ fracamente em $H^{1}\left(\mathbb{R}^{N}\right)$ e fortemente em $L_{\text {loc }}^{q}\left(\mathbb{R}^{N}\right)$, para todo $2<q<2^{*}$. Além disso, por (3.12), $w_{0} \neq 0$. Agora, de (3.11), vale a seguinte identidade

$$
\int_{\mathbb{R}^{v}}\left(\nabla w_{n} \nabla w_{0}+V\left(h_{n} x+h_{n} y_{n}\right) w_{n} w_{0}\right) d x=\int_{\mathbb{R}^{N}} w_{n}^{p} w_{0} d x .
$$

Pelo Lema de Fatou e por $\left(V_{7}\right)$ temos

$$
\int_{\mathbb{R}^{: v}}\left(\left|\nabla w_{0}\right|^{2}+V_{0} w_{0}^{2}\right) d x<\int_{\mathbb{R}^{N}} w_{0}^{\mathrm{r}+1} d x
$$

Seja $\theta>0$ o número que verifica

$$
I_{0}\left(\theta w_{0}\right)=\max _{t \geq 0} I_{0}\left(t w_{0}\right)
$$

De $(3.13)$, segue que $\theta \in(0,1)$, logo

$$
\begin{aligned}
c_{0} & \leq I_{0}\left(\theta w_{0}\right)=\left(\frac{1}{2}-\frac{1}{p+1}\right) \theta^{p+1} \int_{\mathbb{R}^{N}} w_{0}^{p+1} d x<\left(\frac{1}{2}-\frac{1}{p+1}\right) \int_{\mathbb{R}^{N}} w_{0}^{p+1} d x \\
& \leq\left(\frac{1}{2}-\frac{1}{p+1}\right) \liminf _{n \rightarrow \infty} \int_{\mathbb{R}^{N}} w_{n}^{p+1} d x=\liminf _{n \rightarrow \infty} c_{n}=c_{0},
\end{aligned}
$$

o que é um absurdo, provando assim o lema.

Observação 3.6. Segue da demonstraçăo do Lema 3.5 que se $\left(h_{k} y_{h_{k}}\right)$ converge para $x_{0}$, então $V\left(x_{0}\right)=V_{0}$, isto é, $x_{0}$ é minimo global de $V$.

De fato, considere

$$
w_{k}(x)=v_{h_{k}}\left(x+y_{h_{k}}\right)
$$

Como na demonstração do Lema $3.5, w_{k} \rightarrow \bar{w}$ fracamente em $H^{1}\left(\mathbb{R}^{N}\right)$, com $\bar{u}$ sendo uma função não-negativa. Agora,

$$
\int_{\mathbb{R}^{N}}\left(\nabla w_{k} \nabla \bar{w}+V\left(h_{k} x+h_{k} y_{h_{k}}\right) w_{k} \bar{w}\right) d x=\int_{\mathbb{R}^{N}} w_{k}^{p} \bar{w} d x
$$

Sabendo que

$$
\int_{\mathbb{R}^{N}}\left(|\nabla \bar{w}|^{2}+V\left(x_{0}\right) \bar{w}^{2}\right) d x \leq \liminf _{n \rightarrow \infty} \int_{\mathbb{R}^{N}}\left(\nabla w_{k} \nabla \bar{w}+V\left(h_{k} x+h_{k} y_{h_{k}}\right) w_{k} \bar{w}\right) d x,
$$

encontramos

$$
\int_{\mathbb{R}^{N}}\left(|\nabla \bar{w}|^{2}+V\left(x_{0}\right) \bar{w}^{2}\right) d x \leq \int_{\mathbb{R}^{N}} \bar{w}^{p+1} d x
$$


Desse modo, se $V_{0}<V\left(x_{0}\right)$ então

$$
\int_{\mathbb{R}^{N}}\left(|\nabla \bar{w}|^{2}+V_{0} \bar{u}^{2}\right) d x<\int_{\mathbb{R}^{N}} \bar{w}^{p+1} d x .
$$

Repetindo o mesmo argumento empregado na etapa final da demonstração do Lema 3.5 obtemos uma contradição com o nível minimax $c_{0}$, o que conclui a verificação da observação.

Lema 3.7. A seqüência $w_{k}$ converge fortemente para $\bar{w}$ em $H^{1}\left(\mathbb{R}^{N}\right)$.

Demonstração: Primeiramente, por $\left(V_{7}\right)$,

$$
\begin{aligned}
c_{k}=I_{k}\left(v_{k}\right)-\frac{1}{p+1} I_{k}^{\prime}\left(v_{k}\right) v_{k} & \geq\left(\frac{1}{2}-\frac{1}{p+1}\right) \int_{\mathbb{R}^{N}}\left(\left|\nabla v_{k}\right|^{2}+V_{0} v_{k}^{2}\right) d x \\
& =\left(\frac{1}{2}-\frac{1}{p+1}\right) \int_{\mathbb{R}^{N}}\left(\left|\nabla w_{k}\right|^{2}+V_{0} w_{k}^{2}\right) d x
\end{aligned}
$$

onde $v_{k}=v_{h_{k}}$. Pelo Lema 3.3,

$$
\limsup _{k \rightarrow \infty}\left(\frac{1}{2}-\frac{1}{p+1}\right) \int_{\Omega^{N}}\left(\left|\nabla w_{k}\right|^{2}+V_{0} w_{k}^{2}\right) d x \leq c_{0} .
$$

Agora, pelo Lema 3.5 e Observação $3.6, \bar{u}$ satisfaz

$$
-\Delta \bar{w}+V_{0} \bar{w}=\bar{w}^{p} \text { em } \mathbb{R}^{N}, \bar{w}>0 \text { em } \mathbb{R}^{N}
$$

o qual juntamente com o Lema de Fatou e (3.14) implicam

$$
\begin{aligned}
c_{0} & \leq I_{0}(\bar{w})=I_{0}(\bar{w})-\frac{1}{p+1} I_{0}^{\prime}(\bar{w}) \bar{u} \\
& =\left(\frac{1}{2}-\frac{1}{p+1}\right) \int_{\mathbb{R}^{N}}\left(|\nabla \bar{w}|^{2}+V_{0} \bar{w}^{2}\right) d x \\
& \leq \liminf _{k \rightarrow \infty}\left(\frac{1}{2}-\frac{1}{p+1}\right) \int_{\mathbb{R}^{N}}\left(\left|\nabla w_{k}\right|^{2}+V_{0} w_{k}^{2}\right) d x \\
& \leq \limsup _{k \rightarrow \infty}\left(\frac{1}{2}-\frac{1}{p+1}\right) \int_{\mathbb{R}^{N}}\left(\left|\nabla w_{k}\right|^{2}+V_{0} w_{k}^{2}\right) d x \\
& \leq c_{0} .
\end{aligned}
$$

Portanto,

$$
\lim _{k \rightarrow \infty} \int_{\mathbb{R}^{N}}\left(\left|\nabla w_{k}\right|^{2}+V_{0} w_{k}^{2}\right) d x=\int_{\mathbb{R}^{N}}\left(|\nabla \bar{w}|^{2}+V_{0} \bar{w}^{2}\right) d x
$$


Em conseqüência do Lema 3.7 e da imersão de $H^{1}\left(\mathbb{R}^{N}\right)$ em $L^{2^{*}}\left(\mathbb{R}^{N}\right)$, decorre que

$$
\int_{|x| \geq R}\left|w_{k}\right|^{2^{*}} d x \rightarrow 0, \text { quando } R \rightarrow \infty \text {, uniformemente em } k \text {. }
$$

De fato, seja $c>0$ dado. De $\bar{w} \in H^{1}\left(\mathbb{R}^{N}\right)$ e $H^{1}\left(\mathbb{R}^{N}\right) \subset L^{2^{*}}\left(\mathbb{R}^{N}\right)$, existe $R_{0}>0$ tal que

$$
\int_{|x| \geq R}|\bar{u}|^{2 *} d x<\frac{\epsilon}{2} ; \quad \forall R \geq R_{0}
$$

Por outro lado, como $w_{k} \rightarrow \bar{w}$ fortemente em $H^{1}\left(\mathbb{R}^{N}\right)$, novamente pela imersão de Sobolev; $u_{k} \rightarrow \bar{w}$ fortemente em $L^{2^{*}}\left(\mathbb{R}^{N}\right)$. Logo, existe $k_{0} \in \mathbb{N}$ tal que

$$
\int_{\mathbb{R}^{N}}\left|w_{k}-\bar{w}\right|^{2} d x<\frac{\epsilon}{2} \quad \forall k \geq k_{0}
$$

Por $(3.16)$ e (3.17), para todo $k \geq k_{0}$ e $R \geq R_{0}$ temos

$$
\int_{|x| \geq R}\left|w_{k}\right|^{2^{*}} d x \leq \int_{\mathbb{R}^{N}}\left|u_{k}^{\prime}-\bar{w}\right|^{2^{*}} d x+\int_{|x| \geq k}|\bar{w}|^{2^{*}} d x<\epsilon
$$

Agora, como $w_{k}$ é uma subsolução de $\Delta u+c(x) u=0, \operatorname{com} c(x)=w_{k}^{p \cdots 1}(x)$, pelo Teorema $8.17 \mathrm{~cm} \mathrm{[8],} \mathrm{segue} \mathrm{que}$

$$
\max _{B_{1}(P)} w_{k} \leq C\left(\int_{B_{2}(P)}\left|w_{k}\right|^{2^{*}} d x\right)^{\frac{1}{2^{*}}}
$$

no qual $P$ é um ponto arbitrário e $C$ depende somente de $N$ e da constante que limita $\left\|u_{\kappa^{\prime}}\right\|_{L^{2^{*}}\left(B_{2}(P)\right)}$. Assim, dado $\delta>0$, existe $R>0$ tal que

$$
\max _{|x| \geq R} w_{k}(x)<\delta, \quad \forall k \geq k_{\ominus}
$$

Seja $p_{k} \in \mathbb{R}^{N}$ um máximo local de $u_{k}$. Como

$$
-\Delta w_{k}\left(p_{k}\right)+V\left(h_{k} p_{k}+h_{k} y_{k}\right) w_{k}\left(p_{k}\right)=w_{k}^{p}\left(p_{k}\right)
$$

pelo Princípio do Máximo Forte, $w_{k}\left(p_{k}\right) \geq\left(V_{0}\right)^{\frac{1}{p-1}}$. Logo, escolhendo $\delta=\frac{1}{2}\left(V_{0}\right)^{\frac{1}{p-1}}$, existe $R_{0}>0$ tal que

$$
w_{k}(x)<\delta, \quad \forall k \geq k_{0}, \quad|x| \geq R_{0}
$$

De onde segue que $p_{k} \in \bar{B}_{R_{0}}(0)$. Afirmamos que $p_{k}$ é o único máximo local (portanto global) de $w_{k}$. A prova dessa afirmação faz uso de um importante resultado de Gidas-NiNirenberg [7] sobre simetria de soluções e do seguinte lema de Ni-Takagi [9]. 
Lema 3.8. Seja $g \in C^{2}\left(\overline{B_{R}}\right)$ uma função radial satisfazendo $g^{\prime}(0)=0$ e $g^{\prime \prime}(r)<0$ para $0 \leq r \leq R$. Então, existe $\delta>0$ tal que se $f \in C^{2}\left(\overline{B_{R}}\right)$ satisfaz $\nabla f(0)=0$ e $\|g-f\|_{c^{\prime 2}\left(\overline{B_{R}}\right)}<\delta$, tem-se $\nabla f \neq 0$ para $x \neq 0$.

Demonstração: Pelo Teorema Fundamental do Cálcilo e por $\nabla f(0)=0$, tem-se:

$$
\frac{\partial f}{\partial x_{j}}(x)=\int_{0}^{1} \nabla \frac{\partial f}{\partial x_{j}}(t x) d t . x
$$

Fintão,

$$
\frac{\partial f}{\partial x_{j}}(x)=\int_{0}^{1} \nabla \frac{\partial g}{\partial x_{j}}(t x) d t \cdot x+\int_{0}^{1} \nabla\left(\frac{\partial f}{\partial x_{j}}-\frac{\partial g}{\partial x_{j}}\right)(t x) d t . x .
$$

Mas $g$ é radial, isto ć, $g(x)=g(|x|)$; logo, alguns cálculos levam a

$$
\nabla \frac{\partial g}{\partial x_{j}}(t x) d t . x=g^{\prime \prime}(t x) x_{j}
$$

Daí, se $\nabla f(x)=0$, tem-se

$$
-\left(\int_{0}^{1} g^{\prime \prime}(t x) d t\right) t^{2} x_{j}=\int_{0}^{1} \nabla\left(\frac{\partial f}{\partial x_{j}}-\frac{\partial g}{\partial x_{j}}\right)(t x) d t . x, \text { para cada } j=1, \ldots, N .
$$

Agora, seja $\alpha:=\min \left|g^{\prime \prime}\right|$, então $\alpha>0$ se $0 \leq r \leq R$. Logo,

$$
\begin{aligned}
\alpha^{2} x_{j}^{2} & \leq\left|\left(-\int_{0}^{1} g^{\prime \prime}(t x) d t\right) x_{j}\right|^{2} \\
& =\left(\int_{0}^{1} g^{\prime \prime}(t x) d t\right)^{2} x_{j}^{2} \\
& =\left|\int_{0}^{1} \nabla\left(\frac{\partial f}{\partial x_{j}}-\frac{\partial g}{\partial x_{j}}\right)(t x) d t \cdot x\right|^{2} \\
& \leq\left|\int_{0}^{1} \nabla\left(\frac{\partial f}{\partial x_{j}}-\frac{\partial g}{\partial x_{j}}\right)(t x) d t\right|^{2}|x|^{2}
\end{aligned}
$$

donde segue, ao somar-se todos os termos variando $j$ de 1 a $N$, que

$$
\alpha^{2}|x|^{2} \leq N^{2} \sup \left|D^{2}(f-g)\right||x|^{2}
$$

Portanto, se $\|f-g\|_{C^{2}\left(\overline{B_{R}}\right)}<\frac{\alpha^{2}}{N^{2}}$, então necessariamente $|x|=0$.

Aplicando os argumentos utilizados no Capítulo 1 , a seqüência $\left(w_{k}\right)$ converge para $\bar{w}$ no sentido $C_{l o c}^{2}$. Por Gidas-Ni-Nirenberg [7], $\bar{w}$ tem um único máximo em zero, é radialmente simétrica $\mathrm{e}$

$$
\bar{w}(x),|\nabla \bar{w}(x)|=O\left(e^{-|x|} /|x|^{(N-1) / 2}\right)
$$


quando $|x| \rightarrow \infty$. Assim, zero ć o máximo global de $\bar{w}$. Portanto, pelo Lema 3.8, wi não possui outro máximo, a não ser o zero, o que verifica a afirmação.

Recordando que $w_{k}(x)=v_{k}\left(x+y_{h_{k}}\right)$ e que $v_{k}(x)=u_{h_{k}}\left(h_{k} x\right)$, temos que $u_{h_{k}}$ maximizase em $x_{k}=h_{k} p_{k}+h_{k} y_{h_{k}}$, no qual converge para $x_{0}$, pois $\left(p_{k}\right)$ é limitada, $h_{k} \rightarrow 0 \mathrm{e}$ $h_{k} y_{h_{k}} \rightarrow x_{0}$. De onde segue que

$$
\lim _{x \rightarrow \infty} V\left(x_{k}\right)=V_{0}
$$

Finalmente, para provar o decaimento exponencial de $u_{u_{k}}$, necessitamos de um resultado envolvendo $w_{k}$.

Lema 3.9. Existem constantes $C, M>0$ tais que.

$$
w_{k} \leq C \exp ^{-M|x|}, \quad \forall x \in \mathbb{R}^{N}
$$

Demonstração: $\operatorname{De}(3.19)$, existe $R_{0}>0$ tal que

$$
w_{k}(x) \leq \frac{1}{2}\left(V_{0}\right)^{\frac{1}{p-1}}, \text { para todo }|x| \geq R_{0} .
$$

Fixemos $\phi(x)=C \exp ^{-M i x \mid} \operatorname{com} M^{2}<\frac{1}{2}\left(V_{0}\right)^{\frac{1}{p-1}}$ e $w_{k} \leq C \exp ^{-M|x|}$, para todio $|x|=R_{i j}$.

Note que

$$
\Delta \phi \leq M^{2} \phi \text {, para todo } x \neq 0
$$

Definamos $\phi_{h}=\phi-w_{k}$. Por $(3.21)$ e $(3.22)$ temos

$$
\left\{\begin{array}{l}
-\Delta \phi_{h}+\frac{V_{0}}{2} \phi_{h} \geq 0,|x| \geq R_{0} \\
\phi_{h} \geq 0, \quad|x|=R_{0} \\
\lim _{|x| \rightarrow \infty} \phi_{h}=0
\end{array}\right.
$$

O Princípio do Máximo Forte implica que

$$
\phi_{h} \geq 0, \text { para todo }|x| \geq R_{0}
$$

$\log 0$

$$
w_{k} \leq \phi=C \exp ^{-N|x|} \text {, para todo }|x| \geq R_{0} .
$$

Como $R_{0}$ é arbitrário, concluimos a demonstração do Lema 3.9. 
Consequentemente, usando a definição de $u_{k}$ e o Lema 3.9 obtemos

$$
u_{k}(x) \leq C \exp \left(-M\left|\frac{x-h_{k} y_{k}}{h_{k}}\right|\right)
$$

De onde segue

$$
u_{k}(x) \leq C \exp \left(-M\left|\frac{x-x_{k}}{h_{k}}\right|\right)
$$

$\operatorname{com} x_{k} \rightarrow x_{0}$, quando $k \rightarrow \infty, e, V\left(x_{0}\right)=\inf _{x \in \mathbb{X}^{V}} V(x)$ 


\section{Apêndice A}

\section{Diferenciabilidade do Funcional}

\section{Associado}

Definição A.1. Seja $I: U \rightarrow \mathbb{R}$ onde $U$ é um subconjunto aberto de um espaço de Banach $X$. O funcional I possui derivada de Gateaux $G \in X^{\prime}$ em $u \in U$ se, para todo $h \in X$,

$$
\lim _{t \rightarrow 0} \frac{1}{t}[I(u+t h)-I(u)-G t h]=0 .
$$

A derivada de Gateaux em u é denotada por $I^{\prime}(u)$.

Proposição A.2. Se I possui derivada de Gateaux contínua em $U$ então $I \in C^{\mathrm{l}}(U, \mathbb{R})$.

Definição A.3. Seja $I \in C^{1}(U, \mathbb{R})$. O funcional I possui derivada segunda de Gateaux $L \in \mathcal{L}\left(X, X^{\prime \prime}\right)$ em $u \in U$ se, para todo $h, v \in X$,

$$
\lim _{t \rightarrow 0} \frac{1}{t}\left(I^{\prime}(u+t h)-I^{\prime}(u)-L t h\right) v=0 .
$$

A derivada segunda de Gateaux de $I$ em u é denotada por $I^{\prime \prime}(u)$.

Usando o Teorema do Valor Médio pode-se provar a seguinte proposição.

Proposição A.4. Se I possui derivada segunda de Gateaux contínua em $U$ então $I \in$ $C^{2}(U, \mathbb{R})$.

Proposição A.5. Seja $\Omega$ um aberto do $\mathbb{R}^{N}$ e seja $1<p<\infty$. Os funcionals

$$
I(u):=\int_{\Omega}|u|^{p+1} d x, \quad J(u):=\int_{\Omega}\left|u^{+}\right|^{p+1} d x
$$

são de classe $C^{2}\left(L^{p+1}(\Omega), \mathbb{R}\right)$ e

$$
I^{\prime}(u) h_{\iota}=(p+1) \int_{\Omega}|u|^{p-1} u h d x, \quad J^{\prime}(u) h=(p+1) \int_{\Omega}\left|u^{+}\right|^{p} h d x .
$$


Demonstração: Iremos considerar apenas o funcional $I$, a demonstração para o fuıcional $J$ é similar.

Existência da derivada de Gateaux. Sejam $u, h \in L^{p+1}(\Omega)$. Dado $x \in \Omega$ e $0<|t|<1$, pelo Teorema do Valor Médio, existe um $c \in(0,1)$ tal que

$$
\begin{aligned}
\frac{\| u(x)+\left.t h(x)\right|^{p+1}-|u(x)|^{p+1} \mid}{|t|} & =(p+1)|u(x)+\operatorname{cth}(x)|^{p}|h(x)| \\
& \leq(p+1)\left[|u(x)|+\left.|h(x)|\right|^{p}|h(x)| .\right.
\end{aligned}
$$

A desigualdade de Hölder implica que

$$
[|u(x)|+|h(x)|]^{p}|h(x)| \in L^{1}(\Omega) .
$$

E segue do Tcorema de Lebesgue que

$$
I^{\prime}(u) h=(p+1) \int_{\Omega}|u|^{p-1} u h d x .
$$

Continuidade da derivada de Gateaux. Seja $f(u):=(p+1)|u|^{p-1} u$. Suponha que $u_{n} \rightarrow u$ em $L^{p+1}$, então $f\left(u_{n}\right) \rightarrow f(u)$ em $L^{q}$ onde $q:=(p+1) / p$. Pela desigualdade de Hölder, obtenos

$$
\left|\left(I^{\prime}\left(u_{n}\right)-I^{\prime}(u)\right) h\right| \leq\left|f\left(u_{n}\right)-f(u)\right|_{q}|h|_{p+1},
$$

e então

$$
\left\|I^{\prime}\left(u_{n}\right)-I^{\prime}(u)\right\| \leq\left|f\left(u_{n}\right)-f(u)\right|_{q} \rightarrow 0, \text { quando } n \rightarrow \infty .
$$

Existência da derivada segunda de Gateaux. Sejanı $u, h, v \in L^{p+1}$. Dado $x \in \Omega$ e $0<|t|<1$, pelo Teorema do Valor Médio, existe um $c \in(0,1)$ tal que

$$
\begin{aligned}
\{[f(u(x)+t h(x))-f(u(x))] v(x)|/| t \mid & =(p+1) p|u(x)+\operatorname{cth}(x)|^{p-1}|h(x)||v(x)| \\
& \leq(p+1) p[|u(x)|+|h(x)|]^{p-1}|h(x)||v(x)|
\end{aligned}
$$

A desigualdade de Hölder implica que

$$
[|u(x)|+|h(x)|]^{p-1}|h(x)||v(x)| \in L^{1}(\Omega) .
$$

E segue do Teorema de Lebesgue que

$$
I^{\prime \prime}(u) h v=(p+1) p \int_{\Omega}|u|^{p-1} h v d x .
$$


Continuidade da derivada segunda de Gateaux. Seja $g(u):=(p+1) p|u|^{p-1}$. Suponha que $u_{n} \rightarrow u$ em $L^{p+1}$, então $g\left(u_{n}\right) \rightarrow g(u)$ em $L^{r}$ onde $r:=(p+1) /(p-1)$. Pela desigualdade de Hölder, obtemos

$$
\mid\left[\left(I^{\prime \prime}\left(u_{n}\right)-I^{\prime \prime}(u)\right) h|v| \leq\left|g\left(u_{n}\right)-g(u)\right|_{r}|h|_{p+1}|v|_{p+1}\right.
$$

e entào

$$
\left\|_{i}^{\prime \prime}\left(u_{n}\right)-I^{\prime \prime}(u)\right\| \leq\left|g\left(u_{n}\right)-g(u)\right|_{r} \rightarrow 0, \text { quando } n \rightarrow \infty
$$

Em conseqüência das imersões de Sobolev e da Proposição A.5, temos seguinte corolário. Corolário A.6. Se $1<p \leq \frac{N+2}{N-2}$ e $N \geq 3$ então os funcionais $I$ e $J$ são de classe $C^{2}\left(H_{0}^{1}(\Omega), \mathbb{R}\right)$. 


\section{Apêndice B}

\section{Teorema do Passo da Montanha}

Lema B.1. Sejam $X$ um espaço de Hilbert real, $I \in C^{2}(X, \mathbb{R}), c \in \mathbb{R}, \epsilon>0$. Considere o conjunto $A_{s}=\{u \in X: I(u) \leq s\}$. Suponha que

$$
\left\|I^{\prime}(u)\right\| \geq 2 \epsilon, \quad \forall u \in I^{-1}([c-2 \epsilon, c+2 \epsilon]) .
$$

Então existe uma função $\eta \in C(X, X)$ tal que:

(i) $\eta(u)=u$, se $I(u) \in[c-2 \epsilon, c+2 \epsilon]$,

(ii) $\eta\left(A_{c+\varepsilon}\right) \subset A_{c-\epsilon}$.

\section{Demonstração: Sejam}

$$
\begin{gathered}
A:=\{u \in X: c-2 \epsilon \leq I(u) \leq c+2 \epsilon\} \\
B:=\{u \in X: c-\epsilon \leq I(u) \leq c+\epsilon\} \\
g(u):=\frac{\|u-A\|}{\|x-A\|+\|x-B\|} .
\end{gathered}
$$

Então $g=1 \mathrm{em} B, g=0 \mathrm{em} X \backslash A$ e $g$ é localmente Lipschitz contínua em $X$. Seja $f$ um campo vetorial localmente Lipschitz contínuo definido por

$$
f(u):= \begin{cases}-g(u)\|\nabla I(u)\|^{-2} \nabla I(u), & u \in A, \\ 0, & u \in X \backslash A\end{cases}
$$

É claro que $\|f(u)\| \leq(2 \epsilon)^{-1}$ em $X$. Para cada $u \in X$, o problema de Cauchy

$$
\begin{aligned}
\frac{d}{d t} \psi(t, u) & =f(\psi(t, u)) \\
\psi(0, u) & =u
\end{aligned}
$$




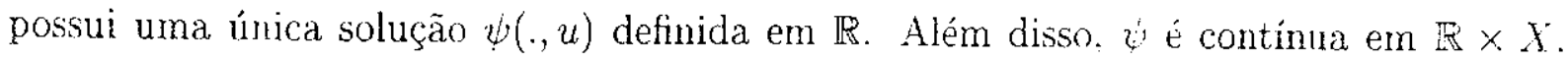
Então a aplicação $\eta(u):=\psi(2 \epsilon, u)$ satisfaz $(i)$.

Como

$$
\begin{aligned}
\frac{d}{d t} I(\psi(t, u) & =\left\langle\nabla I(\psi(t, u)), \frac{d}{d t} \psi(t, u)\right\rangle \\
& =\langle\nabla I(\psi(t, u)), f(\psi(t, u)\rangle \\
& =-g(\psi(t, u)\rangle
\end{aligned}
$$

temos que $I(\psi(., u))$ é não crescente. Seja $u \in A_{c+\epsilon}$. Se existir $t \in[0,2 c]$ tal que $I(\psi(t, u))<c-\epsilon$, então $I(\psi(2 \epsilon, u))<c-\epsilon$ e (ii) está satisfeito. Se

$$
\psi(t, u) \in B, \forall t \in[0,2 \epsilon]
$$

entĩo por (B.2),

$$
\begin{aligned}
I(\psi(2 \epsilon, u)) & =I(u)+\int_{0}^{2 \epsilon} \frac{d}{d t} I(\psi(t, u)) d t \\
& =I(u)-\int_{0}^{2 \epsilon} g(\psi(t, u)) d t \\
& \leq c+\epsilon-2 \epsilon=c-\epsilon
\end{aligned}
$$

c (ii) está satisfeito.

Teorema B.2. Sejcm $X$ um espaço de Hilbert real e $I \in C^{2}(X, \mathbb{R})$. Suponha que $I(0)=0$ e

$\left(\phi_{1}\right)$ Existem constantes $\rho, \alpha>0$ tais que $b:=\left.\inf I(u)\right|_{\partial B_{\rho}} \geq \alpha$,

$\left(\phi_{2}\right)$ Existe um $e \in X \backslash \overline{B_{\rho}}$ tal que $I(e) \leq I(0)$.

Entäo, para cada $€>0$, existe um $u \in X$ tal que

a) $c-2 \epsilon \leq I(u) \leq c+2 \epsilon$,

b) $\left\|I^{\prime}(u)\right\|<2 \epsilon$,

onde

$$
c:=\inf _{\gamma \in \Gamma} \max _{t \in[0,1]} I(\gamma(t))
$$

com

$$
\Gamma:=\{\gamma \in C([0,1], X): \gamma(0)=0 . \gamma(1)=e\}
$$


Demonstração: A hipótese $\left(\phi_{1}\right)$ implica

e então

$$
b \leq \max _{t \in[0,1]} I(\gamma(t))
$$

$$
b \leq c \leq \max _{t \in[0,1]} I(t e)
$$

Suponhamos que, para algum $\epsilon>0$, a conclusão do teorema não é satisfeita. Podemos assumir entào que

$$
c-2 \epsilon \geq I(0) \geq I(e)
$$

Pela definição de $c$, existe $u$ m $\gamma \in \Gamma$ tal que

$$
\max _{t \in[0,1]} I(\gamma(t)) \leq c+\epsilon .
$$

Considere $\beta:=\eta \circ \gamma$, onde $\eta$ é dado pelo Lema B.1. Por (B.3) e pela parte (i) do Lema anterior,

$$
\beta(0)=\eta(\gamma(0))=\eta(0)=0,
$$

e analogamente $\beta(1)=c$, e então $\beta \in \Gamma$. Segue de (B.4) c (ii) do Lema B.1 que

$$
c \leq \max _{t \in[0, \mathbb{1}]} I(\beta(t)) \leq c-\epsilon
$$

o que é uma contradição.

Para provar que $c$ é um valor crítico de $I$, nós precisamos do seguinte rosultado de compacidade.

Definição B.3. Sejam $X$ um espaço de Banach, $I \in C^{1}(X, \mathbb{R})$. Uma seqüêrcia $\left(u_{n}\right)$ é uma seqüiência de Palais-Smale no nível c (seqüência $(P S)_{c}$ ) relacionada a I se

$$
I\left(u_{n}\right) \rightarrow c, \quad I^{\prime}\left(u_{n}\right) \rightarrow 0
$$

Dizemos que o funcional I satisfaz a condição $(P S)_{c}$ se toda seqüência $(P S)_{c}$ possui urna subseqüência convergente em $X$.

Teorema B.4. (Ambrosetti-Rabinowitz) Suponha que as hipóteses do Teorema B.2 sejam válidas. Se I satisfaz a condição $(P S)_{c}$, então c é valor crítico de $I$.

Demonstração: $\quad$ O Teorema B.2 implica a existência de uma seqüência $\left(u_{n}\right) \subset X$ satisfazendo (B.5). Pela condiçāo $(P S)_{c}$, a seqüência $\left(u_{n}\right)$ possui uma subseqüência convergindo para $u \in X$. Entāo $I(u)=c$ e $I^{\prime}(u)=0$. Portanto $c$ é valor crítico de $I$. 


\section{Apêndice C}

\section{Princípio Variacional de Ekeland}

Teorema C.1. (Princípio Variacional de Ekeland) $\operatorname{Seja}(M, d)$ um espaço métrico completo. Seja $f: M \rightarrow(-\infty, \infty]$ uma função semicontínua inferiormente, limitada inferiormente ef $f \neq \infty$. Dado $\epsilon>0$, tome $u \in M$ tal que

$$
f(u) \leq \inf _{M} f+\epsilon
$$

Então existe $v \in M$ tal que

$$
\begin{array}{r}
f(v) \leq f(u) \\
d(u, v) \leq 1
\end{array}
$$

$\because$ para cada $w \neq v$ em $M$

$$
f(w)>f(v)-\epsilon d(v, w)
$$

Demonstração: Primeiro definamos uma relação de ordem em $M$ da seguinte forma

$$
w \leq v \Leftrightarrow f(w)+\epsilon d\left(v, u_{i}\right) \leq f(v)
$$

É imediato que as propriedades da relação de ordem são satisfeitas.

(i) (reflexiva) $u \leq u$;

(ii) (anti-simétrica) $u \leq v$ e $v \leq u$ implica que $u=v$;

(iii) (transitiva) $u \leq v$ e $v \leq w$ implica $u \leq u$; para todo $u, v, w \in M$.

Definamos agora uma seqüência $\left(S_{n}\right)$ de subconjuntos de $M$ da seguinte forma: começando $\operatorname{com} u_{0}=u$, seja

$$
S_{0}=\left\{w \in M: w \leq u_{0}\right\}
$$


e escolhemos $u_{1} \in S_{0}$ tal que $f\left(u_{1}\right) \leq \inf _{S_{0}} f+1$. Por indução definimos

$$
S_{n}=\left\{w \in M: w \leq u_{n}\right\}
$$

e escolhemos $u_{n+1} \in S_{n}$ tal que $f\left(u_{n+1}\right) \leq \inf _{S_{n}} f+\frac{1}{(n+1)}$.

Claramente $S_{n+1} \subset S_{n}$ quando $u_{n-1} \leq u_{n}$. $S_{n}$ é fechado: seja $x_{j} \in S_{n}$ com $x_{j} \rightarrow$ $x \in M$. De $x_{j} \in S_{n}$ temos que $f\left(x_{j}\right) \leq f\left(u_{n}\right)-\epsilon d\left(x_{j}, u_{n}\right)$. Passando o limite e usando a semicontinuidade inferior de $f$ e a continuidade de $d$ temos que $x \in S_{n}$. Agora. Se $w \in S_{n+1}, w \leq u_{n-1} \leq u_{n}$ o que implica

$$
\epsilon d\left(u, u_{n+1}\right) \leq f\left(u_{n+1}\right)-f(u) \leq \inf _{S_{n}} f+\frac{1}{(n+1)}-\inf _{S_{n}} f=\frac{1}{(n+1)}
$$

$\operatorname{logo} d\left(w, u_{n+1}\right) \leq \frac{1}{\epsilon(n+1)}$ e entāo diàmetro $S_{n+1} \leq \frac{1}{\epsilon(n+1)}$, isto é, diâmetro $S_{n} \rightarrow 0$ quando $n \rightarrow \infty$. O fato de $M$ scr completo implica que

$$
\bigcap_{n=1}^{\infty} S_{n}=\{v\}
$$

para algum $v \in M$. Em particular, $v \in S_{0}$, isto é: $v \leq u_{0}=u$ e então

$$
d(u, v) \leq \epsilon^{-1}(f(u)-f(v)) \leq \epsilon^{-1}\left(\inf _{M} f+\epsilon-\inf _{M} f\right)=1
$$

Para obter (C.3) ć suficiente mostrar que $w \leq v$ implica que $w=v$. Se $w \leq v$ então, para cada $n \in \mathbb{N}$

$$
w \leq u_{n}
$$

$\log 0 w \in \bigcap_{n \in \mathbb{N}} S_{n}$ e, por $(\mathrm{C} .4), w=v$

Teorema C.2. Sejam $K$ um espaço métrico compacto, $K_{0} \subset K$ um conjunto fechado, $\mathrm{Y}$ um espaço de Banach, $\varphi \in C\left(K_{0}, X\right)$ e defina o espaço métrico completo $M$ por

$$
M=\left\{g \in C(K, X): g(s)=\varphi(s), \text { ses } s \in K_{0}\right\}
$$

com a distância usuald. Seja $I \in C^{1}(X, \mathbb{R})$ e defina

$$
c=\inf _{g \in M} \max _{s \in K} I(g(s)), \quad c_{1}=\max _{\varphi\left(K_{0}\right)} I .
$$

Se c $>c_{1}$ então para cada $\epsilon>0$ e cada $f \in M$ tal que

$$
\max _{s \in K} I(f(s)) \leq c+\epsilon
$$


existe um $v \in X$ tal que

$$
\begin{gathered}
c-\epsilon \leq I(v) \leq \max _{s \in K} I(f(s)), \\
d(v, f(K)) \leq \epsilon^{1 / 2}, \\
\left|I^{\prime}(v)\right| \leq \epsilon^{1 / 2} .
\end{gathered}
$$

Demonstração: Sem perda de generalidade, podemos assumir que

$$
0<\epsilon<c-c_{1}
$$

Seja $f \in M$ satisfazendo a condição (C.5). Definimos a função $\Phi: M \rightarrow \mathbb{R}$ dada por

$$
\Phi(g)=\max _{s \in K} I(g(s))
$$

e então $c=\inf _{M} \Phi>c_{1}$. Para mostrar que $\Phi$ ć contínua, usaremos a continuidade uniforme de $I$ em $g(K)$.

Pelo Teorema C.1, existe $h \in M$ tal que

$$
\begin{gathered}
\Phi(h) \leq \Phi(f) \leq c+\epsilon \\
\max _{s \in K}|h(s)-f(s)| \leq \epsilon^{1 / 2}
\end{gathered}
$$

$e$

$$
\Phi(g)>\Phi(h)-\epsilon^{1 / 2} d(h, g)
$$

sompre que $g \in M$ e $g \neq h$. Na verdade, o teorema estará provado se mostrarmos a existîncia de algum $s \in K$ tal que

$$
\begin{gathered}
c-\epsilon \leq I(h(s)) \\
\left|I^{\prime}(h(s))\right| \leq \epsilon^{1 / 2},
\end{gathered}
$$

isto é,

$$
\left\langle I^{\prime}(h(s)), v\right\rangle \geq-\epsilon^{1 / 2}
$$

sempre que $v \in X$ e $|v|=1$. Suponhamos, por contradição, que para cada $s \in S$, onde

$$
S=\{s \in K: c-\epsilon \leq I(h(s))\}
$$


existam $\delta_{s}>0, v_{s} \in X$ com $\left|v_{s}\right|=1$ c uma bola aberta $B_{s}$ em $K$ contendo $s$ tal que, para $t \in B_{s} \circ u \in X \operatorname{com}|u| \leq \delta_{s}$, tenhamos

$$
\left\langle I^{\prime}(h(t))+u, v_{s}\right\rangle<-\epsilon^{1 / 2} .
$$

Como $S$ ó compacto, existe uma subcobercura finita $B_{s_{1}}, \ldots, B_{s_{k}}$ de $S$ e definamos $\psi_{j}$ : $K \rightarrow[0,1]$ por

$$
\begin{aligned}
& \psi_{j}(t)=\frac{d\left(t, B_{s_{j}}^{c}\right)}{\sum_{i=1}^{k} d\left(t, B_{s_{i}}^{c}\right)}, \text { se } t \in \bigcup_{i=1}^{k} B_{s_{i}} \\
& \psi_{j}(t)=0, \quad \text { se } t \in K \backslash \bigcup_{i=1}^{k} B_{s_{i}} .
\end{aligned}
$$

Finalmente, considere $\delta=\min \left(\delta_{s_{1}}, \ldots, \delta_{s_{k}}\right), \psi: K \rightarrow[0,1]$ uma função contínua tal que

$$
\begin{aligned}
\psi(t) & =1, \text { se } c \leq I(h(t)) \\
& =0, \text { se } I(h(t)) \leq c-\epsilon .
\end{aligned}
$$

e seja $g \in C(K, X)$ definida por

$$
g(t)=h(t)+\delta \psi(t) \sum_{i=1}^{k} \psi_{j}(t) v_{s_{j}} .
$$

Segue de (C.6) que para $t \in K_{0}$

$$
I(h(t))=I(\varphi(t)) \leq c_{1}<c-\epsilon
$$

e então $\psi(t)=0$. Na verdade, para $t \in K_{0}, g(t)=h(t)=\varphi(t), \log 0 g \in M$.

O Teorema do Valor Médio e (C.8) implicam que, para cada $t \in S$, existe um $0<\tau<1$ tal que

$$
\begin{aligned}
I(g(t))-I(h(t)) & \left.=\left\langle I^{\prime}\left(h(t)+\tau \delta \psi(t) \sum_{j-1}^{k} \psi_{j}(t) v_{s_{j}}\right), \delta \psi(t) \sum_{j=1}^{k} \psi_{j}(t) v_{s_{j}}\right)\right\rangle \\
& \left.=\delta \psi(t) \sum_{j=1}^{k} \psi_{j}(t)\left\langle I^{\prime}\left(h(t)+\tau \delta \psi(t) \sum_{j=1}^{k} \psi_{j}(t) v_{s_{j}}\right), v_{s_{j}}\right)\right\rangle \\
& \leq-\epsilon^{1 / 2} \delta \psi(t) .
\end{aligned}
$$

Se $t \notin S, \psi(t)=0$ e $I(g(t))=I(h(t))$. Se $\bar{t}$ é tal que $I(g(\bar{t}))=\Phi(g)$, obtemos

$$
I(h(t)) \geq I(g(\bar{t})) \geq c
$$


e então $\bar{t} \in S$ e $\psi(t)=1$. Por (C.9) obtemos

$$
I(g(\bar{t}))-I(h(\bar{t})) \leq \epsilon^{1 / 2} \delta .
$$

Em particular,

$$
\Phi(g)+\epsilon^{1 / 2} \delta \leq I(h(\bar{t})) \leq \Phi(h)
$$

$\log \circ \neq h$. Mas, pola definição de $g$ temos $d(g, h) \leq \delta$ e então

$$
\Phi(g)+\epsilon^{1 / 2} d(g, h) \leq \Phi(h)
$$

contrariando (C.7) e completando assim a demonstração.

Corolário C.3. Sobre as hipóteses do Teorema C.2, para cada seqüência $\left(f_{k}\right)$ em $M$ tal que

$$
\max _{K} I\left(f_{k}\right) \rightarrow c
$$

existe uma seqüencia $\left(v_{k}\right)$ em $X$ tal que

$$
\begin{gathered}
I\left(v_{k}\right) \rightarrow c \\
d\left(v_{k}, f_{k}(K)\right) \rightarrow 0 \\
\left|I^{\prime}\left(v_{k}\right)\right| \rightarrow 0,
\end{gathered}
$$

$q u a n d o k \rightarrow \infty$.

Demonstração: Basta definirmos $\epsilon_{k}=\max _{K} I\left(f_{k}\right)-c$ se $\max _{K} I\left(f_{k}\right)-c>0, \epsilon_{k}=1 / k$ se $\max _{K} I\left(f_{k}\right)-c \leq 0$ e aplicarmos o Teorema C.2 para $\epsilon_{k}$ e $f_{k}$, para todo $k \in \mathbb{N}^{*}$. 


\section{Apêndice $D$}

\section{Lema de Lions}

Lema D.1. (P.L. Lions, 1984) Suponha $\left(u_{m}\right)$ limitada em $H^{1}\left(\mathbb{R}^{N}\right)$ e seja $R>0$ lal que

$$
\liminf _{m \rightarrow \infty} \sup _{y \in \mathbb{R}^{N}} \int_{B_{R}(y)}\left|u_{m}\right|^{2} d x=0
$$

Então $u_{m} \rightarrow 0$ em $L^{q}\left(\mathbb{R}^{N}\right)$, onde $2<q<2^{*}$.

Demonstração: Para qualquer $y \in \mathbb{R}^{N}$ e $q \in\left(2,2^{*}\right)$, temos pela desigualdade do Hölder e pela imersão de Sobolev que

$$
\|u\|_{L^{q}\left(B_{R}(y)\right)} \leq C\|u\|_{L^{2}\left(B_{R}(y)\right)}^{1-\lambda}\|u\|_{H^{1}\left(B_{R}(y)\right)}^{\lambda}
$$

onde $\lambda=\frac{(q-2)}{2 q} N$ e $C$ depende de $q, N$ e $R$ mas independe de $y$. Suponha $\lambda q \geq 2$, isto $q \geq \frac{4}{N}+2=\bar{q}$. Então

$$
\int_{B_{R}(y)}|u|^{q} d x \leq C^{q}\|u\|_{L^{2}\left(B_{R}(y)\right)}^{q(1-\lambda)}\|u\|_{H^{1}\left(\mathbb{R}^{N}\right)}^{\lambda q-2} \int_{B_{R}(y)}\left(|\nabla u|^{2}+|u|^{2}\right) d x .
$$

Escolhendo uma família de bolas $\left\{B_{R}\left(y_{i}\right)\right\}$ cuja união cobre $\mathbb{R}^{N}$ e tal que cadá ponto de $\mathbb{R}^{N}$ está contido em no máximo $k$ bolas, obtemos

$$
\|u\|_{L^{q}\left(\mathbb{R}^{N}\right)} \leq k C^{q} \sup _{y \in \mathbb{R}^{N}}\left(\int_{B_{R}(y)} u^{2} d x\right)^{q(1-\lambda)}\|u\|_{H^{1}\left(\mathbb{R}^{N}\right)}^{\lambda q} .
$$

Tomando $u=u_{m}$, temos por (D.1) e (D.3) que o Lema é válido para $q \geq \bar{q}$.

Se $2<q<\bar{q}$, então $q=2 \lambda+\bar{q}(1-\lambda)$ para algum $\lambda \in(0,1)$. Pela desigualdade de Hölder;

$$
\|u\|_{L^{q}\left(\mathbb{R}^{N}\right)}^{q} \leq\|u\|_{L^{2}\left(\mathbb{R}^{N}\right)}^{\lambda}\|u\|_{L^{q}\left(\mathbb{R}^{N}\right)}^{(1-\lambda)}
$$

Tomando $u=u_{m} \mathrm{em}$ (D.4), o resultado então segue pelo caso anterior. 


\section{Referências Bibliográficas}

[1] AMBROSETTI, A.; RABINOWTTZ, P.H. Dual variational methods in critical point theory and applications, J. Funct. Anal. v.14, p.349-381, 1973.

[2] BREZIS, H. Analyse Fonctionelle, Théorie et applications, Paris: Masson, 1987, $233 \mathrm{p}$.

[3] DING, W.Y.; NI, W. M. On the existence of positive entire solutions of a semilinear elliptic equation, Arch. Rat. Mech. Anal., v.91, p.283-308 1986,.

[4] FELMER, P. L.; DEL PINO, M. Local mountain passes for semilinear elliptic problems in unbounded domais, Calc. Var., v.4, p.121-137, 1996.

[5] FELMER, P. L.; DEL PINO, M. Multi-peaks bound states of nonlinear Schrödinger equations, Ann. Inst. Poincaré, Anal. Nonlineaire, v.15, p.127-149, 1998.

66] FLOER, A.; WEINSTEIN, A. Nonspreading wave packets for the cubic Schrödinger equations with a bounded potencial, J. Funct. Anal. v.69, p.397-408, 1986.

[7] GIDAS, B.; NI, W. M.; NIRENBERG, L. Symmetric of positive solutions of nonlinear equations in $\mathbb{R}^{N}$, Advances in Math. Suppl. Studies 7A, Math. Anal. and Applications, Part A. New York: Academic Press, p.369-402, 1981.

[8] GILBARD, D.; TRUDINGER, N. S. Elliptic partial differencial equations of second order, 2.ed, Springer-Verlag, New York, 513 p, 1983.

[9] NI, W. M.; TAKAGI, I. On a shape on least-energy solutions to a semilinear Neumann problem, Comm. on Pure and Appl. Math., v.44, 1991, p.819-851,

[10] NI, W. M.; TAKAGI, I. Locating the peaks of least-energy solutions to a semilinear Neumann problem, Duke Math. J., v.70, p.247-281, 1993. 
[11] NI, W. M.; WFI, J. On the location and profile of spike-layer solutions to singularly perturbed semilinear Dirichlet problems, Comm. on Pure and Appl. Math., v.48, p. $731-768,1995$.

[12] OH, Y. J. Existence of semi-classical bound states of nonlinear Schrödinger equations with potencial on the class $(V)_{a}$, Comm. Partial Diff. Eq., v.13, p.1499-1519, 1988.

[1:3] OH, Y. J. Corrections to Existence of semi-ciassical bound states of nonlinear Schrödinger equations with potencial on the class $(V)_{a}$, Comm. Partial Diff. Eq., v.14, p.833-834, 1989.

[14] RABINOWITZ, P. H. Minimax methods in critical point theory with applications to differential equations, Conference Board of the Mathematical Sciences, Regional Conference Series in Mathematics, A.VS, v.65, 1986, 100p.

[15] RABINOWITZ, P. H. A Note on a semilinear elliptic equation on $\mathbb{R}^{N}$, Differentia! Equations, p.307-317, 1991.

[16] RABINOWITZ, P. H. On a class of nonlinear Schrödinger equations, Z. Angew Math. Phys., v.43, p.270-291, 1992.

[17] WANG, X. On concentracion of positive bound states of nonlinear Schröndiger equatiuns. Commun. Math. Phys., v.153, p.229-244, 1993.

[13] WILLEM, M. Minimax theorems, Birkhäuser, Boston, 1996, 159p. 